University of Louisville

ThinkIR: The University of Louisville's Institutional Repository

\title{
A structural equation analysis of the relationships between PTSD, depressive symptoms and suicidal ideation : is general strain theory a viable framework for explaining suicide risk among correctional staff?
}

Wm. Clay Johnson

University of Louisville

Follow this and additional works at: https://ir.library.louisville.edu/etd

Part of the Criminology Commons, Other Psychology Commons, and the Social Psychology and Interaction Commons

\section{Recommended Citation}

Johnson, Wm. Clay, "A structural equation analysis of the relationships between PTSD, depressive symptoms and suicidal ideation : is general strain theory a viable framework for explaining suicide risk among correctional staff?" (2018). Electronic Theses and Dissertations. Paper 3015.

https://doi.org/10.18297/etd/3015

This Master's Thesis is brought to you for free and open access by ThinkIR: The University of Louisville's Institutional Repository. It has been accepted for inclusion in Electronic Theses and Dissertations by an authorized administrator of ThinkIR: The University of Louisville's Institutional Repository. This title appears here courtesy of the author, who has retained all other copyrights. For more information, please contact thinkir@louisville.edu. 
A STRUCTURAL EQUATION ANALYSIS OF THE RELATIONSHIPS BETWEEN PTSD, DEPRESSIVE SYMPTOMS AND SUICIDAL IDEATION: IS GENERAL STRAIN THEORY A VIABLE FRAMEWORK FOR EXPLAINING SUICIDE RISK AMONG CORRECTIONAL STAFF?

\author{
By
}

Wm. Clay Johnson

B.A., University of Louisville, 2010

A thesis submitted to the College of Arts and Sciences of the University of Louisville in partial fulfillment of the requirements for the degree of

Masters of Science in Criminal Justice

Department of Criminal Justice University of Louisville

Louisville, Kentucky

August 2018 

A STRUCTURAL EQUATION ANALYSIS OF THE RELATIONSHIPS BETWEEN PTSD, DEPRESSIVE SYMPTOMS AND SUICIDAL IDEATION: IS GENERAL STRAIN THEORY A VIABLE FRAMEWORK FOR EXPLAINING SUICIDE RISK AMONG CORRECTIONAL STAFF?

\author{
By \\ Wm. Clay Johnson \\ B.A. University of Louisville, 2010 \\ A Thesis Approved on: \\ July 25, 2018 \\ by the following Thesis Committee: \\ Dissertation Director \\ Kirstin Swartz, Ph.D. \\ George E. Higgins, Ph.D. \\ Matthew Logan, Ph.D.
}




\section{DEDICATIONS}

This is dedicated to my loving wife (whose patience apparently has no bottom)

for still marrying me, despite my spending far too much time away from her and with this

paper. 


\section{ACKNOWLEDGMENTS}

First, I would like to thank my academic mentor and chair, Dr. Kristin Swartz, for her guidance and understanding throughout this writing process. I would also like to thank Dr. Swartz and her research assistants for allowing me to use the data they worked so hard to collect. I would also like to thank Dr. George Higgins for taking his time to teach me an analytic technique I had no business using. Finally, I would like to thank Dr. Matthew Logan for serving on my committee.

Clay Johnson 


\title{
ABSTRACT \\ A STRUCTURAL EQUATION ANALYSIS OF THE RELATIONSHIPS BETWEEN PTSD, DEPRESSIVE SYMPTOMS AND SUICIDAL IDEATION: IS GENERAL STRAIN THEORY A VIABLE FRAMEWORK FOR EXPLAINING SUICIDE RISK AMONG CORRECTIONAL STAFF?
}

\author{
Wm. Clay Johnson \\ July $25^{\text {th }}, 2018$
}

There is a large body of evidence, which suggests PTSD is strongly related to increased suicide risk, however, some studies suggest the true nature of their relationship is better explained through the development of depression due to the effects of PTSD. While a majority of the studies examining this relationship focus only on general populations, a growing body research suggests that high-risk occupations such as police, firefighters and correctional staff are developing PTSD at rates as high as 8 to 10 times that of the rate of these general samples. This study, then, aims to fill a small gap in this nascent research into PTSD and suicide among high-risk occupations. Guided by a General Strain Theory framework, this study uses structural equation modeling to examine whether depression is capable of mediating the effect PTSD has on suicide risk. The results of this study support the use of GST as a viable framework for examining these relationships within high-risk samples, revealing depression mediated a negative direct relationship exhibited by PTSD on suicidal ideation. This suggests that while PTSD may still play a role in increased suicidal behavior, the main effect PTSD displays 
on increased suicidal thought is instead a product of the depression developed alongside other PTSD symptoms. These findings have implications for the treatment of PTSD and the handling of workplace-related trauma for individuals in high-risk occupations, especially correctional facilities. 


\section{TABLE OF CONTENTS}

Chapter 1: Introduction.........................................

The Current Study........................................4-5

Chapter 2: Literature Review.....................................6-39

Posttraumatic Stress Disorder.................................6-25

DSM-IV Criteria................................... $7-9$

PTSD Prevalence......................................9

PTSD and Comorbidity............................ 9-10

PTSD and Trauma.....................................10-17

Age, Gender, and PTSD .............................15-17

PTSD and High-Risk Samples........................18-23

Correctional Staff and PTSD.........................23-25

Depression and PTSD.....................................26-30

Age, Gender, and Other Factors...................27-29

Depression and Correctional Staff....................29-30

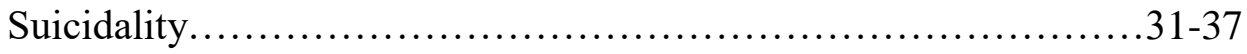

Suicidality in Veterans..............................34-35

Suicidality in Firefighters/E.M.T.'s.................35

Suicidality in Law Enforcement.....................36

PTSD, Depression, and Suicidality ...................... 37 
Gaps in Existing Research................................38-39

Chapter 3: Theoretical Framework..................................40-65

Sources of Strain.............................................. $41-42$

Forms of Coping ............................................. $43-44$

Types of Strain.............................................44-46

Characteristics of Strains related to Delinquency.................46-51

Negative Affect's Mediating Effect............................51-54

Empirical Status of GST ......................................54-63

Tests of Emotions as Mediators...........................57-59

Coping as a Mediator....................................60

GST and Anti-Social Coping............................60-63

The Current Study ...........................................63-65

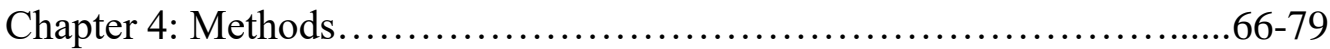

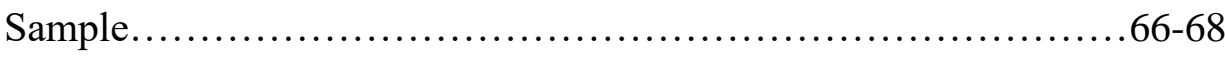

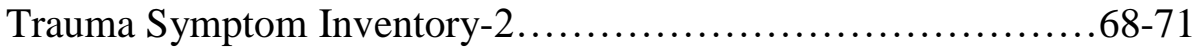

Analysis \& Variables: Structural Equation Modeling..............72

Independent Variable: PTSD .................................72-76

Mediating Variable: Depression...............................76-78

Dependent Variable: Suicidal Ideation..........................78-79

Chapter 5: Results................................................. $80-92$

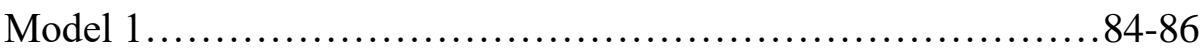

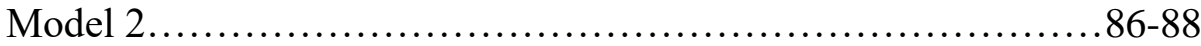

Model 3.................................................... $88-90$ 
Model 4.

$90-92$

Chapter 6: Discussion........................................93-108

Limitations to the Current Study...........................98-101

Implications of Future Research............................101-106

Conclusion.............................................106-108

References.................................................... 109-121

Curriculum Vitae..............................................122 


\section{LIST OF FIGURES}

Figure 1: TSI-2 Factors and Subfactors.............................78

Figure 2: Descriptive Statistics Table................................88

Figure 3: Bivariate Correlations Tables.............................91-92

Figure 4: Confirmatory Factor Analysis............................. 95

Figure 5: Direct Effects Model.....................................98

Figure 6: Indirect Effects Model.....................................99

Figure 7: Direct and Indirect Effects Model..........................101 


\section{CHAPTER 1: INTRODUCTION}

Prisons in the United States are overcrowded, underfunded, and understaffed. It is no surprise, then, that studies into the mental health of employees at correctional facilities, from security personnel to medical staff, consistently find that correctional staff report higher prevalence rates of mental disorders, specifically Posttraumatic Stress Disorder (PTSD) and depression than the general population (Denhof \& Spinaris, 2013, 2016; Spinaris, Denhof \& Kellaway, 2012; Swartz, French, \& Roberts, 2017). These findings regarding corrections staff are not unique in the sense that other occupations that involve first and second-hand exposure to violence and death, such as military veterans, (Ilgen et al., 2010; Hellmuth, Stappenbeck, Hoerster, \& Jakupcak, 2012), police officers (Marmar et al., 2006; Robinson, Sigman, \& Wilson, 1997), EMTs (Marmar, Weiss, Metzler, Ronfeldt, \& Foreman, 1996), and firefighters (Boffa et al., 2016; Stanley, Hom, Spencer-Thomas, \& Joiner, 2017), have all found evidence that high levels of exposure to on-duty trauma are linked to higher levels of PTSD symptoms. Interestingly, there are very few studies examining the effects of working within a correctional institution on its staff (either security or non-security), despite those few studies that do exist indicating that they display having higher prevalence rates of PTSD than all other "high-risk" occupations except for military veterans. 
The existing research on PTSD and depression prevalence among correctional staff suggests that it is the combination of many small stressors related to the nature of working in corrections that contributes to these high rates of severe mental health issues. For example, Denahof, Spinaris, and Morton (2014) mentioned stressors such as the repeated exposure to the aftermath of graphic violence and inmate suicides; the constant state of hypervigilance from being outnumbered by potentially violent offenders; and/or the workplace cultural requirement to not show emotion, empathy, or weakness. When combined with a lack of access to positive coping skills and resources or negative coping skills (e.g. substance use), they may result in a serious decline of mental health.

Another major difference that should be noted between correctional staff and other high-risk occupations is the consistency with which correctional staff have to deal with these occupational stressors. To elaborate, while firefighters display significantly higher rates of PTSD than the general population, (Boffa et al., 2016; Stanley et al., 2017), firefighters also working as EMT's riding with victims have been found to report slightly higher rates of PTSD than individuals working as firefighters only. This suggests that length of exposure to a stressor can increase the risk of mental illness (Marmar et al., 1996). This could mean that another reason correctional staff see higher rates of both PTSD and depression may be due to their entire shift being full of exposure to noxious stimuli. That is, correctional staff may feel constantly on edge or uneasy due to being outnumbered by inmates. It should be noted that this stress would not just be on the security staff then, as non-security employees like clerical workers, medical staff, and mental health professionals might all feel uneasy or experience other forms of indirect exposure to traumas (e.g. reading explicit details of inmate caseloads or treating injuries 
following an assault). If an individual working in a correctional institution were to fear for their safety and experience some form of direct trauma, whether that be physical violence or even a very violent threat, it is not hard to believe that they might develop hypervigilance or other PTSD-related symptoms. Aside from a gap in the literature about work-related trauma and mental disorders among correctional staff, there is even less research investigating another major problem that suffered across high-risk occupations: suicide risk.

Suicide is a serious enough problem in the general population: According to a 2018 report by the Center for Disease Control, suicide was one of only 3 leading causes of death to see an overall rate increase over the last decade and was the fourth overall leading cause of death for Americans ages 18-65. While the suicide can never be said to have one cause, there is one common circumstance surrounding suicides, reported to the National Violent Death Reporting System (NVDRS) (data collection about the circumstances surrounding all violent deaths in 27 states, including Kentucky), mental health issues. The NVDRS reports that a majority of suicides, specifically, $52.59 \%$ involved circumstances surrounding a recent "mental health problem." This would imply that, while not all suicides are the results of mental health issues, they did play a role, however small, in a majority of the fourth leading cause of death in 2016.

The literature regarding occupations at a high risk for trauma-related metal disorders and suicide supports this, with studies indicating that veterans (Ilgen et al., 2010; Hellmuth et al., 2012), police (Violanti, 2004) and firefighters (Boffa et al., 2015; Stanley et al., 2017) all show higher scores on various measures of suicidality (i.e. both suicidal ideation and suicidal behavior). Beyond simply showing that these occupations 
have high-risk for suicide, they show that this higher risk is directly related to the higher rates of PTSD and depression seen among the samples, although the exact relationships are inconsistent. The only study examining the suicide rates of correctional employees focused specifically on security personnel and was published over 21 years ago (Stack \& Tsoudis, 1997). Still, it found that correctional officers had higher suicide rates than those seen in the general population but did not determine whether this was at all related to PTSD, depression or any mental illness. Instead, the authors speculated that one potential cause for these higher than average suicide rates were from the abnormally high levels of stress that exist in correctional institutions. As suicide is a growing problem, further research is needed to help fill the gaps in the literature surrounding both the relationships between PTSD, depression, and suicidality as well the problem of increased suicide among correctional staff.

\section{The Current Study}

In an effort to fill the existing gaps in the literature, the current study seeks to apply a theoretical framework to the problem of suicide among correctional officers with PTSD. More specifically, this study examines the relationships between PTSD, depression, and suicidality through a General Strain Theory (Agnew, 1992) lens and Structural Equation Modeling is used to analyze these relationships. GST, a general theory of crime suggests that criminal or other antisocial coping behaviors (e.g. suicidality) occur when a negative life event causes a negative emotional state. If an individual lacks the resources or access to positive coping, then they may resort to antisocial coping methods as a way to remove the strain and alleviate the negative state (Agnew, 1992). In applying this to the problem of suicidality among correctional staff, 
this study examines PTSD as an analog for work-related trauma and is considered the "strain." It is then likely that this strain would create a negative emotional state, such as depression, that could then be alleviated through antisocial coping (i.e. suicidal ideation). The analysis of this study then, examines three separate hypotheses: 1) Whether higher rates of PTSD in a sample of correctional staff are related, directly or indirectly, to varying rates of suicidal ideation; 2) Whether the effects of PTSD on suicide risk are instead better explained as PTSD's influence on depression; and 3) Whether General Strain Theory can be used as a viable framework for examining the relationships between PTSD, depression and suicidality. This study aims to fill several gaps in the existing literature on these topics.

The following section, Chapter 2, is a review of the relevant literature, starting with a discussion of Posttraumatic Stress Disorder and its correlates, followed by the same reviews of depression and Suicidality. Following that section is Chapter 3, a discussion of the theoretical framework used to guide this study: General Strain Theory (GST). This chapter not only looks at the original work creating the theory, but also reviews a large body of relevant studies that apply the GST framework. Chapter 4 is a thorough discussion of the methods used in the design and follow-through of the current study, including the sampling and measuring tools used in the final analysis. Chapter 5 discusses the results of the analysis and the final section, Chapter 6, discusses the major implications of these finding 


\section{CHAPTER 2: LITERATURE REVIEW}

\section{Posttraumatic Stress Disorder}

Posttraumatic Stress Disorder (PTSD) is an intense, prolonged psychological, behavioral, and physiological reaction to the exposure to a traumatic event. Recognized as one of the trauma and stressor-related disorders in the Diagnostic and Statistical Manual of Mental Disorders $5^{\text {th }}$ Edition (DSM-V), PTSD and the other disorders in this section are set apart from other closely related disorders (e.g. anxiety or dissociative disorders) because of their distinct diagnostic requirement for exposure to a traumatic or high-stress life event. In this context, trauma exposure is defined as any situation where an individual is the subject of or witness to "actual or threatened death, serious injury, or sexual violence” (APA, 2013, p. 271).

Beyond its requirement for exposure to trauma, PTSD is characterized by intrusive experiences (e.g. vivid nightmares, flashbacks), defensive avoidance of stimuli related to details of the trauma, increased negative physiological reactions (e.g. hypervigilance, insomnia) and significant negative changes to thought patterns and mood, all beginning immediately or shortly after the traumatic experience (APA, 2013). According to a review of the diagnostic criteria of PTSD and other disorders by Young, 
Lareau, and Pierre (2014), there are over 600,000 unique possible symptom combinations an individual with PTSD can exhibit. In other words, there are over a halfmillion ways a person can experience PTSD before considering any of the many potential comorbid disorders. Once comorbid disorders like anxiety and depression are included, the potential symptom combinations reach beyond a quintillion (Young, Lareau, and Pierre, 2014). This can make it difficult for researchers to pinpoint exactly which symptoms are occurring due to PTSD or due to one of its comorbid disorders. For this reason, it is important to both understand that criteria used in diagnosing PTSD and how important each criterion is to a diagnosis of PTSD.

\section{DSM-V Criteria}

The DSM-V provides five distinct criteria that are required in order to qualify for a PTSD diagnosis. Criterion (A) is the most important to the diagnosis of PTSD: The exposure to a traumatic event itself, which includes both direct and indirect exposure to traumas. Although it existed in the previous edition, the DSM-5 was the first time that the APA recognized that "indirect" traumas (i.e. second- or third-hand exposures) were distinct from "direct" traumas (i.e. first-hand exposure) as potential sources of PTSD. These "indirect traumas" include learning that a loved one had experienced a traumatic event through someone else after it occurred (excluding learning of natural death or early death by illness) or experiencing repeated or extreme exposure to the details of a traumatic event (e.g. therapists exposed to details of abuse or police exposed to violent crime scenes) (APA, 2013). Along with updating which specific types of events count as "traumatic" (e.g. limiting third-party death exposure to learning about violent and accidental deaths only), the DSM-V was also the first time that Criterion A specifically 
included repeat and vicarious exposure to explicit details of traumatic events (e.g. therapists' repeated exposure to explicit details of abuse) (APA, 2013). This criterion must be met for an individual to receive a PTSD diagnosis.

The second criterion (B) for diagnosis is the development of at least one intrusive symptom after exposure to the traumatic event. Examples of these intrusive thoughts include involuntary memories of the traumatic event, distressing dreams/nightmares related to the trauma, flashbacks, and/or intense psychological distress or physiological reactions caused by trauma-related cues (APA, 2013). In an attempt to reduce these intrusive symptoms, individuals may begin to avoid internal cues (e.g., thoughts or feelings) or external cues (e.g., places and things) that remind them of their trauma. This newly developed defensive behavior represents the third criterion $(\mathrm{C})$, which is defined by successful or attempted avoidance of memories and thoughts about the traumatic event, or people, places, or things that invoke such memories (APA, 2013). When paired together, these criteria make up the characteristic symptoms of PTSD and are therefore both required to have developed after the trauma exposure in order for there to be a possible diagnosis.

PTSD's Criterion D includes the development of two or more negative changes in thought processes and/or mood-specifically, symptoms like dissociative amnesia related to the trauma, negative attributional style changes, constant negative emotional state, depersonalization, detachment, and anhedonia (APA, 2013). Finally, criterion (E) is considered present when at least two new problems involving increased physiological reactivity (i.e. irritability/angry outbursts, self-destructive behavior, hypervigilance, exaggerated startle response, and problems with sleep or concentration) develop after 
exposure to the traumatic event (APA, 2013). If the minimum requirements of all the criteria are met or exceeded for one month, and significantly impact an individual's functioning in their social, occupational, educational life, a diagnosis of PTSD can be considered (APA, 2013).

\section{PTSD Prevalence}

Among adults in the United States, PTSD has a reported lifetime prevalence of 8.3-8.7\%, a 12- month prevalence of 3.5-4.7\%, and a 6-month prevalence of 3.8\% (APA, 2013; Kessler et al., 1995; Kilpatrick, et al., 2013). Lifetime prevalence rates have been found to be consistent over time independent of DSM diagnostic criteria; studies using outdated DSM criteria have found similar reported lifetime prevalence when compared to studies using DSM-V criteria, around 7.8\% in 1995 (Kessler et al., 1995) to 9\% in 2001 (Marshall et al., 2001).

PTSD prevalence is reported as being significantly higher in both veteran samples (Ilgen et al., 2010; Hellmuth, et al., 2012; Raab, Mackintosh, Gros, \& Morland, 2015; Rytwinski et al., 2013) and those taken from occupations that involve consistent exposure to dangerous or potentially traumatic situations such as police (Marmar et al., 2006; Robinson, Sigman, \& Wilson, 1997), EMTs (Marmar et al., 2006), firefighters (Boffa et al., 2016; Stanley, Hom, Spencer-Thomas, \& Joiner, 2017), and correctional staff (Denhof \& Spinaris, 2013, 2016; Spinaris et al., 2012).

\section{PTSD and Comorbidity}

Individuals with PTSD are roughly $80 \%$ more likely to meet the full criteria for at least one other disorder compared to those without PTSD (APA, 2013). Some of the most common disorders experienced comorbidly with PTSD include depression in 
general (Cougle et al., 2009a, 2009b; Dorrington et al., 2014; Marshall et al., 2001; Stevens et al.,2013) and high-risk samples (Chen, Yoon, Harford, \& Grant, 2017; Denhof \& Spinaris, 2013, 2016; Rytwinski, Scur, Feeny, \& Youngstrom, 2013; Spinaris et al., 2012); alcohol/substance abuse in both general samples (Neupane, Bramness, \& Lien, 2017; Smith et al., 2016) and samples of high-risk populations (Chen et al., 2017; Hellmuth et al., 2012); and Traumatic Brain Injury (especially in military samples) (APA, 2013; Kessler et al., 1995).

One common focus of studies examining the relationships between PTSD and its most common comorbid disorders was an attempt to understand why these disorders occurred together at such a high frequency. One consistent finding in this body of literature is that the development of PTSD after trauma exposure is heavily related to later development of other disorders, like substance abuse (Breslau, 2002) and depression (Breslau, 2009; Breslau, Davis, Peterson, \& Schultz, 2000; Kessler et al., 2003; Wang et al., 2000). Before these potential causal effects can be more thoroughly examined, however, it is important to understand the different risk factors that are recognized as being related to the initial PTSD development. The section below discusses these risk factors and their differing effects on the development of PTSD across general and highrisk samples.

\section{PTSD and Trauma}

The most important risk factor associated with PTSD is also its defining diagnostic criterion: the exposure to traumatic event. Studies that have examined the prevalence of traumatic exposure have found that it is nearly ubiquitous, with $90 \%$ of individuals in general population samples reporting at least one event that would qualify 
as "traumatic" under the DSM-V's criterion A (Kilpatrick et al., 2013). In some high-risk samples, such as correctional officers, the prevalence of traumatic exposure can reach nearly 100\% (Denhof \& Spinaris, 2012; 2013). However, since prevalence rates of PTSD are much lower, this begs the question of what factors are involved in determining whether an individual develops PTSD as a reaction to a traumatic experience. Although traumatic experiences are common, fewer than $10 \%$ of individuals react to that trauma by developing PTSD in samples from the general population (APA, 2013; Breslau, 2009; Kessler et al., 1995). This may have to do with factors involving the trauma itself (i.e. type and severity), individual characteristics (i.e. age and sex, personality characteristics) or access to coping resources and skills (i.e. familial and peer support). The following section discusses how specific characteristics of the traumatic exposure itself influence rates of PTSD development.

The "type" of trauma refers to the specific characteristics surrounding a traumatic event, such as whether it was experienced directly or indirectly, assaultive/interpersonal or non-assaultive/accidental. For example, traumatic events such as violent victimization, armed robbery, and rape could be categorized as assaultive or interpersonal traumas; meanwhile, life-threatening illnesses or physical injuries caused in accidents or natural disasters are described as non-assaultive traumas. Along with the cause of the trauma, the "type" of trauma can also refer to whether it is experienced as a single event, a complex trauma, or a chronic or cumulative trauma. Being robbed is a single traumatic event; however, if a robbery also involved a physical assault, the existence of two distinct traumatic elements create a complex trauma, in which multiple traumatic experiences occur during one event. Finally, the last two categories involve repeated exposure to 
traumatic events over long periods of time. Chronic trauma refers instances in which one or more traumatic event is repeatedly and directly experienced over time (e.g. child abuse/neglect). Repeated direct and indirect exposure to trauma can also compound, as has been seen among first responders and their repeated exposure to death and serious physical injury. This trauma builds on itself over time and is referred to as cumulative trauma. Research has found results revealing a complex relationship between these types of trauma and individual differences in who develops PTSD as a response.

Single-event traumas among general samples were found to be most related to PTSD when they were assaultive or interpersonal, especially in cases of sexual assault, torture, or physical assault (with and without a weapon) (Breslau, 2009; Dorrington et al., 2014; Kilpatrick et al., 2013; McNally \& Robinaugh, 2011). The length of recovery time also appears to be longer for those experiencing assaultive or interpersonal traumas. Breslau (2009) specifies that those who experienced direct assaultive traumas had a median recovery time of four years- four times higher than those who experienced indirect traumas. However, indirect or non-assaultive traumas have still been reported as resulting in PTSD.

While the literature indicates single-event traumas of all types (especially assaultive/interpersonal traumas) are related to the development of PTSD, evidence suggests that multiple traumas--whether occurring in the same event (i.e. complex traumas) or during separate events that create a sensitivity to future traumas (i.e. chronic/cumulative traumas) --are much more likely to lead to a development of PTSD (Dorrington et al., 2014; Kilpatrick et al., 2013). For example: A car accident survivors with no injuries (i.e. a single-event, non-assaultive) could develop PTSD, but is 
significantly less likely to do so when compared to a car accident survivor who also sustained life-threatening injuries, had to stay in intensive care, or had injuries requiring minor physical therapy (i.e. a complex, non-assaultive trauma) (Coronas et al., 2008). Dorrington et al. (2014) reported, that among those in their sample who reported at least one trauma exposure $(n=2,180$ of 6,$008 ; 36 \%)$, for every one traumatic experience reported, the percent of the sample reporting PTSD doubled. That is, for single-event exposure, 3.4\% met a PTSD diagnosis; for two cumulative events: 7\%; three or more: 13.3\% (Dorrington et al., 2014). While the measure of cumulative traumas does not specifically measure when the multiple traumas occurred in relation to one another, weakening the ability to say it fully applies to complex traumas, it does show that the number of traumas experienced, regardless of temporality, increase the likelihood of developing PTSD (Dorrington et al., 2014).

Exposure to at least one traumatic event is necessary for- and consistently related toa diagnosis of PTSD. It follows, then, that suffering multiple traumas, regardless of when they occur, would have a higher have risk of a PTSD diagnosis compared to a single traumatic event (Anderson, Geier, \& Cahill, 2016; Dorrington et al., 2014; McNally \& Robinaugh, 2011). This has led researchers to explore how different types of previous traumas can influence the outcome of future exposures (i.e. cumulative trauma). One longitudinal study on cumulative trauma in a sample of adolescents by Cougle and colleagues (2009b) reported that previous direct, assaultive traumas were predictive of a future PTSD response to a second traumatic exposure, whether that exposure was physical assault or witnessing violence. Previous experiences of witnessing violence did not have the same significant effect on either direct or indirect trauma (Cougle et al., 
2009b). The relationship between previous assaultive trauma and higher PTSD rates on a second exposure to any trauma type remains significant even after accounting for PTSD reactions after the first exposure. That is, assaultive traumas are predictive of later PTSD responses, regardless of whether the first trauma elicited PTSD (Cougle et al., 2009b).

Anderson, Geier, and Cahill (2016) found more support for the relationship between multiple traumas and PTSD when investigating a sample of just over 5,000 Black American (i.e. African American and Afro-Caribbean) adults. They reported a significant, direct relationship between a history of incarceration and experiencing more traumatic events: Individuals reporting a history of incarceration also reported experiencing an average of 5.15 traumatic events, compared to the 3.26 events experienced on average by those who had no incarceration history (Anderson et al., 2016). This study also reported that those responding to the survey who reported a history of incarceration were two times more likely to also report a diagnosis of PTSD (12-month and lifetime). Further, experiencing multiple traumas mediated the significance of the relationship between incarceration history and PTSD in those reporting a diagnosis within the last 12-months, but remained significantly related to lifetime PTSD (Anderson et al., 2016). In other words, the effects having been incarcerated on PTSD diagnoses within the last year were better explained as the effects of having experienced multiple traumas while incarcerated. However, this relationship disappears when examining PTSD cases across lifetime. These findings support those of other studies, specifically those which suggest specific traumatic events increases the risk of a PTSD response to later traumas (Cougle et al., 2009b) and that multiple traumas have a significant effect on 
PTSD, independent of previous trauma exposure (Coronas et al, 2008; Dorrington et al., 2014).

To summarize, the main factors related to whether traumatic experiences increase the risk of PTSD among general population samples are whether the traumas are assaultive or interpersonal, whether there were multiple traumas during one event, and/or the existence of previous traumas on to which future traumas might accumulate. The literature suggests that experiencing multiple traumas- regardless of whether complex or cumulative- is the most consistent predictor of PTSD related to the traumatic exposure itself. However, there are other, non-trauma factors that play a role in determining whether trauma exposure develops into PTSD. The following section focuses on some of these non-trauma related risk factors and their role in PTSD development following trauma exposure.

\section{Age, Gender, and PTSD}

Personal demographics such as age and gender have been shown to have an effect on individual reactions to trauma, including the development of PTSD and its symptoms. Age has been associated with an individual's overall risk for traumatic exposures, with the group at the highest risk being those between the ages of 16-25 (Breslau, 2009). Several studies have reported that many of these early traumas can influence the risk that PTSD develops following a second trauma in samples of the general population (Cougle et al., 2009b; McNally \& Robinaugh, 2011; Miller-Graff, Scrafford, \& Rice, 2016). Miller-Graf and colleagues (2016) found that individuals who reported exposure to crimes within their community or reported witnessing violence at a young age were more likely to report PTSD symptoms later in life. Another interesting finding from this study 
was that each of the specific crime types were first experienced at significantly different ages and traumas experienced at younger ages had strong relationships with PTSD reactions (Miller-Graf et al., 2016). Although Miller-Graf et al.’s (2016) findings could be argued to show support for certain traumas displaying stronger connections with PTSD reactions, it absolutely shows support that experiencing violence (i.e. trauma) at younger ages is connected to later developments of PTSD.

Although data on the effects of childhood trauma on high-risk samples (e.g. police, firefighters, EMTS, and/or correctional staff) were scarce, one study did support that this relationship existed beyond the general population. Follette, Polusny, and Milbeck (1994) examined a sample of both mental health $(n=471)$ and law enforcement $(n=87)$ professionals' personal history of childhood sexual abuse were related to higher levels of work related stress, especially when handling cases childhood sexual abuse. They reported a pattern of higher PTSD symptom levels in police officers and mental health professionals who also reported personally experiencing child abuse, regardless of the amount of cases dealing with the subject (Follette, Polusny, \& Milbeck, 1994). In other words, high-risk samples (i.e. police officers) show the same pattern of increased PTSD reactions following childhood trauma.

In regards to gender, studies using samples from general populations have found that women are at a higher risk than men for developing PTSD (APA, 2013; Breslau, 2009). Although one study reported no significant differences between genders (Marshall et al., 2001), most agreed that women displayed PTSD prevalence rates around two times that of men (Breslau, 2009; Coronas et al, 2008; Kilpatrick et al., 2013). While there are several proposed reasons for the relationship, such as higher reported rates of exposure to 
traumas, higher rates of prior traumatic experiences, preexisting anxiety or depression, or sex-based reporting bias, Breslau (2009) found that these proposed explanations fail to explain the gap between the genders. She also reports that in her survey of 18 to 45 -yearold Detroit residents $(n=2,181)$, the only category of trauma in which the gender difference was significant was assaultive traumas. The overall difference in PTSD reported between genders was still significant (women: 13.2\% n=; men: 6\%), supporting other findings.

Unlike PTSD's relationship with age, the relationship between PTSD and gender does not remain the same in high-risk samples, although results are inconsistent between samples. For example, samples of military veterans report insignificant differences between men and women, with men being slightly more likely to report PTSD than women (Ilgen et al., 2010). In a sample of correctional officers Denhof and Spinaris (2016) also found that there was no significant difference between men and women within the sample. However, in a separate sample that included all correctional staff (i.e. all workers, not just security), men were found to be significantly more likely to report experiencing PTSD than women, with $28.7 \%$ of male staffers reporting above threshold PTSD symptoms while only $22.1 \%$ of women within the sample met the same criteria (Denhof \& Spinaris, 2013; Spinaris et al., 2012). Considering the finding discussed in Breslau (2009) which reported only assaultive traumas displayed a significant relationship with gendered PTSD reactions, it is possible the inconsistent finding among correctional staff and officers may be attributable to differing levels of assaultive trauma, although this is speculative. The following section discusses more of the details of highrisk occupations and their relationships with PTSD. 


\section{High-Risk Populations and PTSD}

While much of the research discussed so far has examined samples from the general public, many studies on PTSD focus on occupations that involve an increased risk of potential trauma exposure. Samples from military veterans (Hellmuth et al., 2012; Ilgen et al., 2010; Raab et al., 2015) and other high-risk occupations like police officers (Marmar et al., 2006; Robinson, Sigman, \& Wilson, 1997), EMTs (Marmar et al., 1996), fire fighters (Boffa et al., 2016; Stanley et al., 2017) and correctional staff (Denhof \& Spinaris, 2013, 2016; Spinaris et al., 2012) exhibit PTSD prevalence rates significantly higher than those in the general population. This should make them a focus for future research, as their high risk for PTSD and other psychological disorders indicates a heightened risk of serious maladaptive coping behaviors, such as suicidal thoughts and suicidal behaviors.

Military veterans were the first samples in which early forms of PTSD, known then as "shell-shock" or "war neurosis", were observed (Sher, 2004). It should be no surprise, then, that studies on samples of veterans are consistently seen as reporting higher prevalence of lifetime PTSD (Hellmuth et al., 2012; Ilgen et al., 2010; Raab et al., 2015). A study on psychiatric conditions (including PTSD and depression) and suicide rates among all veterans between the ages of 18 and 80 years old using services provided by the Veterans Health Administration ( $\mathrm{n}=3,291,891)$ reported that the 12-month prevalence of PTSD in the sample was 6.3\% (Ilgen et al., 2010). This is significantly higher than the reported 12-month prevalence for the general population, which ranges from 3.5-4.7\% (APA, 2013; Kessler et al., 1995; Kilpatrick, et al., 2013). As stated above, this study found that unlike the general population, men had slightly higher PTSD 
prevalence than women within the sample, although this may be attributable to the small number of women in the overall sample (Ilgen et al., 2010).

Other studies have examined how previous military experience effects PTSD rates in employees working in high-risk occupations, including firefighters and correctional staff (Boffa et al., 2017; Denhof \& Spinaris, 2013, 2016; Spinaris et al., 2012). Correctional staff who reported previous military experience were studied in both Spinaris, Denhof, \& Kellaway's (2012) and Denhof and Spinaris (2013)'s sample of over 3,500 correctional staffers, as well as in Denhof and Spinaris' (2016) sample of only correctional officers. In the sample of correctional staff, a significant difference was found between those with military experience (30.1\%) and those without (24.7\%) prior to working in corrections (Denhof \& Spinaris, 2013; Spinaris et al., 2012). The 2016 sample of only correctional officers found no significant difference between the groups (Denhof and Spinaris, 2016). It should be noted that while the 2010 veteran study by Ilgen and colleagues found significantly elevated PTSD rates, their prevalence rate was one fifth that found in the sample of correctional staff ( $6.3 \%$ to $30.1 \%$, respectively) with military experience and one fourth for those without prior military experience (24.7\%) (Denahof \& Spinaris, 2013; Spinaris et al., 2012). Samples of firefighters found that those with a military background were significantly more likely to report PTSD symptoms at a clinically elevated level and that military experience was predictive of both higher suicidal ideation and behavior, although this is discussed more later in this section (Boffa et al., 2017).

Despite the higher prevalence rates of PTSD, and in some cases increased suicide risk seen in military veterans working high-risk occupations (Boffa et al., 2017; Ilgen et 
al., 2010), research on the effects of PTSD on suicide within these other high-risk occupations (e.g. police officers, firefighters/EMTs, and correctional staff) is lacking attention in the literature. Studies that examine the rates of PTSD among samples of police, EMTs, and firefighters (i.e. "first responders") often describe prevalence rates that are consistently higher than those seen in the general population. For example, police samples have reported PTSD prevalence rates ranging from 7\% for 6-12 months to $19 \%$ for lifetime (Marmar et al., 2006), nearly two times higher than the general population's prevalence rates, which are estimated to be $3.8-4.7 \%$ for $6-12$ months and $8.3 \%$ over lifetime (Kilpatrick et al., 2013).

Not unlike the general population, however, one study of 635 New York and San Francisco Bay Area police officers found that the levels of PTSD symptomology were significantly related with the frequency and overall number of "critical incidents" (i.e. onduty traumatic events) experienced, an individual's coping skills, and their levels of social support following the incident (Marmar et al., 2006). Beyond this, the most important factor in predicting a PTSD response within the sample was the reporting of a dissociative reaction (e.g. derealization, depersonalization, dissociative amnesia) experienced during the trauma (Marmar et al., 2006). The experience of this "peritraumatic" (i.e. mid-trauma) dissociative event accounted for $21.5 \%$ of the variance in this sample's reported PTSD symptoms, with the total model accounting for 39.7\% of the variance overall (Marmar et al., 2006). This suggests that a peritraumatic reaction to a traumatic event plays a major role in determining which first responders react to traumatic exposure by developing PTSD. 
Another study by Marmar and colleagues (1996) examined the differences between PTSD prevalence in a sample of first responders exposed to the aftermath of a major freeway collapse following an earthquake in California and two control groups of California first responders who were not exposed to that event. While there was no significant difference found in the PTSD measures between the three groups, the overall 12-month prevalence of PTSD throughout the sample was 9\%, around double that found in general samples (Marmar et al., 1996). The study also reported that PTSD rates and instances of peritraumatic dissociation were found to be significantly higher in EMTs/paramedics than in police and firefighters within the sample (Marmar et al., 1996). The authors suggest this may have to do with EMTs' prolonged exposure to the victims following serious accidents, but there is no evidence to support this theory.

Studies examining samples of only firefighters have found results consistent with that on first responders: Boffa and colleagues (2017) reported that $31.8 \%$ of the firefighters in their study ( $\mathrm{n}=893$ ) met or exceeded the threshold for a probable diagnosis of PTSD based on the threshold designed for emergency workers within the Posttraumatic Stress Disorder Checklist-Civilian Version (PCL-C). In another sample, this one of only women firefighters $(n=254)$, the rate of probable diagnosis was also significantly higher than that seen in general samples, with a reported 30-day prevalence rate of $19.7 \%$ (Stanley et al., 2017). It should be noted that while this is far higher than the general population's estimated rates, it is also noticeably lower than the rates seen among the sample including both male and female firefighters. This is consistent with other findings that indicate men report a higher prevalence of PTSD than women in samples from other high-risk occupations (Denhof \& Spinaris, 2013, 2016; Spinaris et al., 
2012; Ilgen et al., 2010). Another interesting finding from this study was that although the majority of traumas related to later PTSD development were due to direct experiences (35.3\%), the second-most pervasive form of traumatic exposure was the repeated exposure to details of trauma through work (31.1\%). While it would be expected that direct traumas would account for a high percentage of PTSD cases in a general sample, the number of individuals who developed PTSD through indirect cumulative trauma seems much higher than one would expect among a general sample.

The problem of indirect cumulative trauma is seen among other studies of highrisk occupations whose findings suggest that "vicarious trauma" can be just as detrimental as directly experienced trauma (Follette, Polusny, \& Milbeck, 1994; Moulden \& Firestone, 2007). Follette et al. (1994), for example, examined the effects of cumulative trauma on a sample of both law enforcement $(n=87)$ and mental health professionals $(\mathrm{n}=471)$. The study reported that mental health professionals who relied on negative coping mechanisms, had high levels of stress in their personal life, and/or reported dissociating or feeling ineffective at providing help while working with child sexual abuse survivors were more likely to report more symptoms of trauma exposure on the Trauma Symptom Checklist-40 (TSC-40). Mental health professionals with a personal history of child abuse or who had a majority of one's caseload involving childhood sexual abuse were not likely to report higher trauma exposure symptoms (Follette et al., 1994). On the other hand, law enforcement professionals who reported negative professional reactions to child abuse cases, high levels of personal stress, and a previous history of childhood abuse (i.e. sexual or physical) were more likely to report significantly elevated trauma symptoms following exposure to child sex abuse cases 
(Follette et al., 1994). Having a majority of one's caseload pertaining to child sexual abuse, negative coping mechanisms and work-related stress were not correlated with trauma symptom levels among law enforcement within this sample (Follette et al., 1994). Despite the high rates of PTSD seen among high-risk samples, especially those in first responder roles such as law enforcement, one group that has lacked focused research are correctional staff. Correctional staff tend to fill several of the roles of first responders, and as the next section shows, their rates of PTSD symptoms reflect this fact.

\section{PTSD and Correctional Staff}

Consistent with the findings in other high-risk work environments, studies on correctional staff (i.e. anyone working within a corrections facility: administrative, security, clerical, and mental health staff) have found significantly higher rates of PTSD than are found in the general population (Denhof \& Spinaris, 2013; Denhof \& Spinaris, 2016; Spinaris et al., 2012). Using a sample of 3,599 correctional staff members, Spinaris et al. (2012) examined which personal and occupational characteristics were significantly correlated with higher rates PTSD. A second analysis was performed a year later examining correlates of depression and the prevalence of comorbid PTSD and depression among the sample (Denhof \& Spinaris, 2013). Both studies reported the 30-day prevalence of PTSD among the sample to be 27\% (Spinaris et al., 2012; Denhof \& Spinaris, 2013). Finally, in a third study of PTSD and depression rates among those with occupations in corrections, Denhof and Spinaris (2016) collected data from 991 Michigan correctional officers and reported a 30-day PTSD prevalence of 33.7\%.

Contrary to findings in the general population, both samples found that rates of PTSD were significantly higher in men (30.5-34.5\%) compared to women (21.7-30.3\%) 
(Denhof \& Spinaris, 2013, 2016; Spinaris et al., 2012). Job title (i.e. security staff or nonsecurity/clerical staff) was also a significant factor, with security staff reporting a $34 \%$ PTSD morbidity rate compared to non-security staff's $21.5-23 \%$ (Denhof \& Spinaris, 2013, 2016; Spinaris et al., 2012). Gender increased the effect job title had as well, with male security staffers being more likely than female staffers to report clinically significant PTSD symptoms (35.8\% to 29.6\%, respectively) (Denhof \& Spinaris, 2013). It should be noted that even non-security correctional staff displayed higher PTSD rates than those reported in the general population- this may be due to non-security staff's high rates of either indirect exposure (e.g. staff witnessing violent assaults, but not experiencing it) or vicarious trauma (e.g. repeated exposure to the aftermath of violent assaults for medical staff; learning details of offenders' crimes or personal trauma history for correctional mental health staff). Other factors include time spent working in corrections, with those recording 10 or more years in corrections also displaying higher levels of PTSD symptoms (40.8\%) compared to those with less than 10 years (25.1\%) (Denhof \& Spinaris, 2013).

Military history among correctional staff was also investigated as a factor in two of the studies. Denhof and Spinaris (2013) originally found results indicating a significant difference in PTSD prevalence between those with military history and those with none (32.6\% to $25.2 \%$, respectively) in the sample of over 3,500 U.S. correctional staffers. However, in a second study using the sample of 991 correctional officers (i.e. security staff) the relationship was not found, with prevalence rates of $34 \%$ in those with military experience and 33.4\% among the non-veteran group (Denhof \& Spinaris, 2016). 
The strongest and most reliable correlation with PTSD in the samples of correctional staff were the number of Violence, Injury or Death (VID) events experienced by an individual. Nearly synonymous with traumatic events, experiencing VID events were very common and were also strongly related to differences in PTSD rates within the sample. All of the participants in the 2012 sample $(n=3,599)$ reported experiencing at least one VID event, with the sample reporting an overall PTSD rate of 27\% (Spinaris, Denhof, \& Kellaway, 2012). This sample also reported that like traumatic experiences, a strong, positive relationship was found between the total number of VID events and types of VID experienced, and the number and severity of PTSD symptoms developed. This also reflects findings in general population samples that suggest multiple traumas are the most consistent predictor of an eventual diagnosis with PTSD. This finding, then, should be of serious concern for correctional staff, considering nearly all of larger sample (i.e. the sample including administrative or clerical staff) reported at least one VID event. Not only does this place them at a higher risk for PTSD, but it may also increase their risk for other psychiatric disorders or maladaptive coping processes, such as suicide. The following section discusses how PTSD may play a role in influencing the development of depression before discussing how the two may interact to increase the risk for suicide.

\section{Depression and PTSD}

It is important to understand the relationship between PTSD and depression in order to examine both why the relationship exists and how experiencing depression might impact an individual with PTSD's ability to cope in a pro-social manner. For example, there is strong evidence that depression experienced by an individual can act as a mediator between PTSD and suicide risk (Cougle, Resnick, \& Kilpatrick, 2009; Marshall 
et al., 2001; Panagioti et al., 2015). Although the nature of relationship between the two disorders is still unclear, there is some evidence that suggests PTSD precedes the development of Major Depression in individuals exposed to trauma (Bebbington et al., 2003; Breslau, 2009; Kessler et al., 2010). The following section provides background information on depression before discussing its relationship with PTSD in greater detail.

The diagnostic criteria to satisfy a diagnosis of depression (i.e. Major Depressive Disorder [MDD] or a Major Depressive Episode [MDE]) are much less rigorous than those of PTSD. Depression's only diagnostic criterion is the requirement that an individual meet a minimum of 5 depressive symptoms from a list of 9 . An individual has to report at least one of two primary symptoms: 1) persistent, depressed mood for most of the day; or 2) a loss of interest/enjoyment in most activities (i.e. anhedonia), for a diagnosis to be considered (APA, 2013). Significant weight/appetite change, sleep disturbances (i.e. insomnia/hypersomnia), slowed speech or movement, fatigue, feelings of worthlessness or inappropriate guilt, problems concentrating, and suicidal ideation/behavior are considered the secondary symptoms, of which three to four must be reported (APA, 2013). If any combination of at least one primary symptom and 3-4 secondary symptoms persists daily (or almost daily) for 2 weeks, cause significant social, occupational and/or familial problems, and not attributable to drugs, alcohol, or another mental illness, the diagnostic criteria is considered fully met (APA, 2013). The reported prevalence for MDD or MDE's in representative samples are reported at a lifetime prevalence of 14.3-16.2\% (Kessler et al., 2003; Stevens et al., 2013) and 12-month prevalence of 6.6-7\%; the average episode lasted around 4 months (APA, 2013; Kessler et al., 2003). Much like PTSD, the prevalence rates of depression vary depending on 
different factors that can influence its development. Some of these factors are discussed in the following sections.

\section{Age, Gender, and Other Factors}

Studies have consistently found that women in general samples display a significantly higher risk of depression than men (Breslau, 2009; Kessler et al. 2003), with some studies reporting that women were two times more likely to develop depression than men (Bebbington et al. 2003). Bebbington and colleagues (2003) suggest that while there is not a clear reason for the sex-related differences, they appear to be social, rather than biological, due to the relationship changing in other samples. One example of this is seen in samples of high-risk occupations, in which the men are consistently more likely to develop depression than the women (Denhof \& Spinaris, 2013, 2016; Spinaris et al., 2012; Ilgen et al., 2010).

These studies also revealed that individuals under the age of 44 were at the highest risk for reporting depression over the past twelve months, compared to individuals in the 45-60+ age group (Bebbington et al., 2003; Kessler et al., 2003). Finally, factors including being a "homemaker," never having married, and having less than 12 years of education were all significantly related to higher reports of depression across 12-months. Life factors such as being unemployed/on disability (12-months), having a history of divorce (lifetime), and having a low-income job (12-months) were also found to be related to "meaningfully elevated" reports of severe Major Depressive Disorder (Kessler et al., 2003). 
Depression is a common response to trauma exposure in both general samples (Breslau, 2009; McCutcheon et al., 2010) and in specific samples such as veterans (Hellmuth et al., 2012; Ilgen et al., 2010; Raab et al., 2015) and other high-risk occupations such as correctional officers (Denhof \& Spinaris, 2013, 2016; Spinaris et al., 2012). McCutcheon et al.'s (2010) analysis of 5,000 twin pairs reported findings that were in line with previous trauma research: Those who reported experiencing assaultive and non-assaultive traumas were more likely to also report higher morbidity rates of depression, panic attacks, or both within the sample (McCutcheon et al., 2010). While both trauma types were significant predictors of either MDD or panic attacks for up to 20 years, assaultive traumas were significant predictors of reporting both disorders over 20 years. (McCutcheon et al., 2010).

Violence, Injury and Death (VID) events reported in samples of correctional staff and correctional officers suggest a relationship between exposure to a VID event and higher reported depression symptoms. Of those reporting at least one VID event within the sample, $27.6 \%$ reported clinically elevated depression symptoms. The relationship between VID events and depression can also arguably be seen vicariously, through the significant relationship found between depression rates and security/non-security jobs and high/low security positions. Those working security staff display higher rates of depression than non-security staff; this might be due to their being at a higher risk of VID exposure due to their direct, constant contact with inmate populations. This same vicarious relationship could be argued when comparing the depression rates of those working in high security levels (which could increase VID risk), to those in low security level positions (Denhof \& Spinaris, 2013; Denhof, Spinaris, \& Kellaway 2012). 
One potential problem with this relationship is the possibility that depression is being indirectly influenced by another factor other than trauma. For example, some findings suggest that developing depression after trauma is more influenced by the preexistence of PTSD (Breslau et al., 2000). Breslau et al.'s (2000) findings showed that those who were exposed to trauma who developed PTSD were at a significantly greater risk for a first-onset of MDD than both those exposed to trauma with no PTSD and those who were not exposed to trauma. Previous cases of PTSD also greatly increased the risk of future MDD episodes compared to groups of non-traumatized individuals and those who did not develop PTSD (Breslau et al. 2000; Breslau \& Peterson, 2010). Breslau (2009) explains that this was evidence that either some individuals may possess an unknown trait that makes them more susceptible to both disorders or PTSD has a strong influence on the development of MDD (Breslau, 2009).

High-risk samples have also demonstrated higher than average levels of depression, especially in those who also reported PTSD symptoms. One sample of veterans reported a depression prevalence rate of $14.6 \%$ across the sample (Ilgen et al., 2010). One longitudinal study of 119 police officers took measurements before training and again after a year on the job and found $77.3 \%$ of the sample reported depression symptoms (Wang et al., 2010). The strongest reported factors related to reported depression symptoms in this study were childhood trauma exposure and feelings of low self-worth at initial collection and feelings of high work-related stress at the 12-month follow-up (Wang et al., 2010). 


\section{Depression and Correctional Staff}

Few studies have been conducted investigating the effects of corrections work on depression rates; however, the findings from the few studies that do exist suggest further research is necessary. Denhof and Spinaris $(2013,2016)$ reported the prevalence of depression symptoms within two different samples of correctional staff, as well as the disorder's relationship with other demographic and occupational factors. The overall prevalence was between 25.7\% (Denhof \& Spinaris, 2013) among all correctional staff (including non-security) and 36.3\% for correctional officers (Denhof \& Spinaris, 2016). These findings are consistent with other reported statistics, such as the higher depression rates seen in security staff (31\%) over non-security staff $(22.1 \%)$, or the findings that show the prevalence rates of low-security groups (31.5\%) are significantly lower than those seen among high-security groups (41.8\%) (Denhof \& Spinaris, 2012. Contrary to other populations, there was no significant difference found in the depression rates between men $(5.2 \%)$ and women $(2.2 \%)$ in the correctional officer sample (Denhof and Spinaris, 2016); meanwhile, the larger sample composed of correctional staff saw significantly higher depression scores among men (28.7\%) than among women (22.1\%) (Denhof and Spinaris, 2012). With correctional staff and other high-risk occupations displaying such high rates of PTSD and depression, reports that suicide rates are higher among these occupations than the general population should come as no surprise. The following section discusses how PTSD and depression might affect an individual's levels of suicidal ideation or behavior. 


\section{Suicidality}

While experiencing PTSD or depression on their own is a major issue to any individual, the fact that these disorders are related to higher rates of suicide through increased suicidal ideation make them a major cause of concern. Given the rising rates of suicide in the U.S. (CDC, 2018), identifying and targeting correlates to increased suicidality should be of importance to researchers. The term "suicidality" refers to two separate constructs that measure whether a person is at a risk for suicide. The first construct, suicidal ideation, refers to thoughts about suicide or which make suicide seem like an option. This can include thoughts such as an individual wishing they were dead, feeling hopeless, or having fantasies about dying/actual thoughts of suicide with no plan. Suicidal ideation is closely related to the second construct of suicidal behavior, which encompasses any behavior done recklessly with the hope/belief that it could lead to death (e.g. taking a large number of pills, drinking and driving) or any actual attempt at suicide, even those in which the individual changes their mind.

Although suicidal ideation is a strong predictor of suicidal behavior, it does not always result in suicidal behavior, suggesting some factors are not enough to influence suicidal behavior through ideation on their own (Panagioti et al. 2015b; Poindexter et al., 2015; Rojas, Bilsky, Dutton, et al., 2017; Stevens et al., 2017). For example, depression influences feelings of hopelessness and worthlessness, making it very closely correlated to suicidal ideation; however, Stevens et al. (2017) reported that even though depression was the most consistently seen disorder among those attempting suicide, it was not found to be a reliable predictor of attempting suicide on its own. This finding suggests that a diagnosis of depression might not be strong enough to cause suicidal behavior on its own, 
but instead acts as a catalyst for suicidal ideation, which mediates the relationship between the other disorders (e.g. PTSD) and suicidal behavior. Further supporting this idea, Stevens et al. (2017) goes on to state that other factors, such as developing multiple psychiatric disorders, likely play a central role in determining which cases of depression result in suicide attempts. Uncovering and treating any of these possible mediating relationships is central to the important task of preventing future suicidal behavior. For this reason, it is especially important to examine these variables within populations that are at a high risk for correlates of suicidality including PTSD and depression (e.g. those high-risk occupations) (Rojas et al., 2017; LeBouthillier et al. 2015; Krysinska, \& Lester, 2010, et al.).

PTSD is consistently seen as being related to antisocial coping mechanisms, such as alcohol use/dependence (Neupane, Bramness, \& Lien 2017; Poindexter et al., 2015) and maladaptive thinking (Breslau et al., 2000; Regambal \& Alden, 2009). It makes sense, then, that PTSD has been linked to higher rates of suicidality in general population samples (Cougle, Resnick, \& Kilpatrick, 2009; Gradus, 2017; LeBouthillier et al. 2015; Marshall et al., 2001; Poindexter et al., 2015; Rojas, et al., 2017; Stevens et al., 2013; Wilcox, Storr, \& Breslau, 2009). A meta-analysis of data from 28 different sources on adolescents with PTSD by Panagioti et al. (2015) reported that if age has a significant effect on PTSD suicidality, it is positive but not strong. Studies using samples of adolescents with PTSD reported a range of 30-80\% also reported suicidal (20-70\% in adults) and while $20-70 \%$ reported suicide attempts (10-40\% in adults) (Panagioti et al., 2015). 
Studies on samples of adolescents and young adults with PTSD consistently found significant differences between genders in relation to suicidality (Panagioti et al., 2015; Wilcox et al., 2009; Langhinrichsen-Rohling, Lewinsohn, Rohde, et al.,1998). These findings suggested that women with PTSD consistently showed higher rates of suicidal ideation, men were significantly more likely to attempt and complete suicide. Findings on rates of suicidality in "general" samples with PTSD were consistent with high-risk samples (with and without PTSD) such as military veterans (Hellmuth et al., 2012; Ilgen et al., 2010) and law enforcement officers (i.e. police and correctional officers) (Tiesman, Hendricks, Bell, \& Amandus, 2010), finding that men were more likely to commit suicide than women. To acknowledge the gap in the current literature, future research should focus on the gender differences in suicide among men and women with PTSD in law enforcement (i.e. police and correctional staff) and working as first responders (i.e. firefighters and EMTs).

One study examined the effects of 27 specific trauma types on suicidal ideation and suicide attempts in individuals with PTSD (LeBouthillier et al., 2015). Using data from the National Epidemiologic Survey of Alcohol and Related Conditions $(\mathrm{N}=34,653)$, LeBouthillier et al. (2015) found results that were consistent with findings on trauma and PTSD. Unsurprisingly, many of the traumas were related to a strong increase in both suicidal ideation and suicide attempts; however, the strongest reported traumas were Childhood Maltreatment (37\% of those with PTSD reporting suicide attempt; $52 \%$ reporting suicidal ideation). Being sexually assaulted/molested/raped (27.8\%; 50.7\%) and those related to "peacekeeping" or war-zone relief $(20.3 \% ; 41.1 \%)$ were the next traumas most strongly related to measures of suicidality (LeBouthillier et al., 2015). 
This same study also examined the effects of experiencing multiple traumas on levels of suicidal ideation and behavior. The analysis revealed that for every one trauma experienced, the rate of suicidal ideation increased by a relative $20 \%$ and suicide attempts increase by $38.9 \%$ (LeBouthillier et al., 2015). This is consistent with previous findings in the trauma literature that suggest experiencing multiple traumas is related to more severe PTSD symptomology (Dorrington et al., 2014; Kilpatrick et al., 2013).

Veteran samples (Ilgen, et al., 2010; Hellmuth et al., 2012) and other high-risk occupations, such as police officers (Tiesman et al., 2010; Wang et al., 2010), correctional officers (Tiesman et al., 2010) and firefighters/EMTs (Boffa et al. 2016; Martin, Tran, Buser, 2017; Stanley et al., 2017) have consistently been found to be related to higher rates of suicidality compared to the general population. Future research should focus on information relating to correctional staff with PTSD and its relationship to suicidality.

\section{Suicidality in Veterans}

Ilgen and colleagues' (2010) study investigated veteran suicides following 7 years of data from the Veteran's Health Administration and National Death Index. The researchers reported findings that $46.8 \%$ of the 7,684 veteran who committed suicide from 1999-2006 had at least one psychiatric disorder at the time; PTSD was present in $11.8 \%(\mathrm{~N}=907)$ of those suicides, while depression was present in $31.2 \%(\mathrm{~N}=2,397)$ (Ilgen et al., 2010). Hellmuth and colleagues' 2012 study examined the specific influence of PTSD's DSM-IV symptom clusters on suicidal ideation and behavior, focusing on the mediating effects of anger, depression, and alcohol use. In a sample of 653 U.S. Iraq/Afghanistan Veterans, they found that the "Numbing" cluster score was the only 
significant direct effect to suicidal ideation making it the only symptom cluster seen to have a direct effect. The effects of the Hyperarousal and Numbing symptom clusters also had an indirect effect through depression (Hellmuth et al., 2012). When taken together these findings seem to suggest that while PTSD is directly related to suicidal ideation and behavior on its own, depression experienced during PTSD plays a mediating role, increasing suicidal ideation.

\section{Suicidality and Firefighters/EMTs}

A study of 3,036 firefighters by Martin, Tran and Buser (2017) reported several different results regarding the relationship between high-risk populations and a lifetime prevalence of reported PTSD and suicidality. First, the authors revealed that although there was no significant elevation of PTSD symptomology found among their sample compared to a provided norm, rates of suicidal ideation, planning, and attempts were all significantly related to PTSD and depression symptom levels (Martin, Tran \& Buser, 2017). Not only were these measures related to PTSD symptom severity, but they were all significantly elevated in the sample. Suicidal ideation (Firefighters: $46.8 \%$; General: 5.6-14.3\%), planning $(19.2 \% ; 3.9 \%)$ and attempts $(15.5 \% ; 1.9-8.7 \%)$ were all significant compared to the levels reported in general samples (Martin, Tran, \& Buser, 2017). This study also reported previous findings that those firefighters who also responded to EMS calls were six times more likely to attempt suicide over their career, yet only one study has been done comparing the groups. This echoes gaps in the research regarding other high-risk samples such as correctional staff, who despite having very high rates of PTSD and depression, have had no studies focused explicitly on the relationship between these factors and their effects suicidality. 


\section{Suicidality and Law Enforcement}

Unfortunately, most research done relating to law enforcement and suicidality did not offer information regarding the effects of PTSD on the sample. The one study reviewed that did include both measures of PTSD symptomology and a measure of suicidality provided results, which were consistent with other results regarding PTSD and suicide in other high-risk occupations. Analysis of Violanti's (2004) sample of 114 police officers found that PTSD symptoms were meaningfully elevated among officers exposed to workplace traumas all types of workplace traumas, with "persons dying" having the strongest effect. The analysis also revealed that individuals with elevated PTSD symptomology also reported higher levels of suicidal ideation and alcohol use (Violanti, 2004).

Another study measuring law enforcement officer occupational deaths over 11 years also reported significantly elevated suicide rates among not only police, but correctional staff as well (Tiesman, et al., 2010). Although this study's relevance is limited due to its lack of PTSD symptom measurements and its inclusion of only those suicides that took place on the job, it still reports that among law enforcement officer suicides made up 10\% $(\mathrm{N}=122)$ of intentional deaths $(\mathrm{N}=1,216)$ over the sample period. Interestingly, the authors also reported that correctional officers were overrepresented in the occupational suicide group, with $29 \%$ of correctional officer intentional occupational deaths being from suicide (Tiesman et al., 2010). While this is the only information on correctional officer suicide rates, these findings are consistent, given the high relationship between correctional staff, PTSD, and depression, and both disorders and suicidality. 


\section{PTSD, Depression, and Suicidality}

Depression is found to be the most common mental disorder among those who attempt suicide (Stevens et al., 2017). One possible explanation for this, however, is depression's seemingly ubiquitous comorbidity leading to it being overrepresented in suicide literature (Kessler et al, 2003). For example, some studies have found that a diagnosis of PTSD alone has a significant effect on suicidality when controlling for depression or depressive symptoms (Gradus, 2017; Cougle, Resnick, \& Kilpatrick, 2009). This might suggest that depression, rather than being predictive of suicidal behavior on its own, instead mediates suicidal behavior in individuals with PTSD by increasing suicidal ideation and other negative cognitive coping mechanisms.

The Ilgen et al. (2010) sample, depression was a diagnosis in $31.2 \%(n=2,397)$ of the 7,684 suicides committed by military veterans from 1999-2006; another military sample found that depression had the strongest direct correlation with suicidal ideation in PTSD (Hellmuth et al., 2013). Other studies the depression-suicidality relationship significant even when controlling for substance use/abuse, anxiety disorders, or PTSD (Hawton, Casañas, Comabella, Haw, \& Saunders, 2013; Wilcox, Storr, \& Breslau, 2009). A meta-analysis of 19 studies on depression and suicidality by Hawton et al. (2013) found a few pronounced risk factors for suicidality in depression, the most salient being: (1) an increased amount of suicide in men with depression over women, (2) a family history of mental disorders increasing suicidality, (3) higher depression severity is direct correlated with higher levels of suicidal tendency and (4) comorbidity with other disorders greatly increased suicidality risks. The following section is a discussion of the gaps in the literature reviewed. 


\section{Gaps in the Existing Research}

This review covered a large body of literature dealing with the Posttraumatic Stress Disorder (PTSD), depression, and increased suicide risk (i.e. suicidality) caused by exposure to various types of workplace trauma. While there is a large body of research examining the relationships between PTSD, depression, and suicidality in samples of military veterans (Hellmuth et al., 2012; Ilgen et al., 2010; McKinney et al., 2017) and firefighters/EMTs (Boffa et al. 2016; Martin et al., 2017; Stanley et al., 2017; Marmar et al., 1996), research on these topics in law enforcement is limited, with the majority of studies examining the effects of correctional staff on PTSD and depression (Denhof \& Spinaris, 2013, 2016; Spinaris et al., 2016) occurring within the last five years. Very few of these studies provide a closer look at the individual influence of PTSD symptoms clusters (Boffa et al., 2016; Hellmuth et al., 2012; Stanley et al., 2017); however, those who did examine the relationships exhibited by the clusters found support for further research. This could include examining both the different representations of PTSD and various forms of depression experienced across various high-risk occupations to determine the existence of common symptom patterns among different subgroups, using clusters to determine if police and firefighter are experiencing the same PTSD. Or, as in Hellmuth et al. (2012), the clusters could be examined to determine individual relationships with negative outcomes of PTSD, such as alcohol or drug abuse and suicide.

The biggest gap in the existing literature on the relationships between PTSD, depression, and suicidality in high-risk occupations is the lack of studies examining the relationship between the heightened prevalence of PTSD and depression among correctional staff and heighten levels of suicidality. The purpose of the following study is 
to fill this gap in the existing literature, using a theoretical framework from Agnew's (1992) General Strain Theory to attempt to explain the relationship between correctional staff's heighted suicidality as a function of the depression symptoms developed along with PTSD. The following section is a review of the relevant GST literature and an explanation as to how it is used in the current study. 


\section{CHAPTER 3: THEORETICAL PERSPECTIVE- GENERAL STRAIN THEORY}

One theoretical lens in which to examine the potential mediating relationship of depression between PTSD and suicidality is that of Agnew's (1992) General Strain Theory (GST). Originally developed as a response to the lack of empirical support for existing strain theories of juvenile delinquency, Agnew's GST (1992) proposed a theory that focuses on individually felt strain and its connection to delinquency. GST defines “strain" as any adverse event or relationship; more specifically, any adversity stemming from the blocking of valued goals, the loss of positively valued stimuli and/or the presentation of negative stimuli. The theory argues that when strain creates a negative affective state in an individual, one likely corrective action or response is crime and delinquency (Agnew, 1992, 2001, 2006; Agnew, Brezina, Wright \& Cullen, 2002). While most people experience strain in some form, few respond with criminal or delinquent coping, tasking GST with explaining individual differences in coping responses to strain (Agnew 1992, 2001, 2006; Agnew, et al., 2002). The remainder of this chapter is dedicated to providing a detailed discussion of GST, a review of the empirical evidence, and concludes with a discussion of how the relationships between PTSD, depression, and suicidality would function within a GST framework. 


\section{Sources of Strain}

In response to some of the major criticisms of classic or traditional strain theories (i.e. that their defined source of strain did not explain a wide enough variety of delinquency or crime), Agnew (1992) expanded strain's definition beyond the blocking of economic goals, identified in classic strain theories (e.g. achieving "the American Dream" or Middle-class status), to include any positively valued goal being prevented and he further added two more broad sources of strain (Merton, 1938; Cohen, 1955; Cloward \& Ohlin, 1960). With the aim of creating a strain theory that could explain as many types of crime and delinquency as possible, Agnew (1992) proposed three broad sources of strain: 1) the blocking of a desired goal; 2) the loss/removal of positive stimuli; and 3) the introduction of a noxious, or negative stimuli. All three types of strain were theorized to have an equal effect on criminality, can be either genuinely experienced or perceived as real, and are not mutually exclusive (i.e. one strain can be described by all three sources) (Agnew, 1992, 2001, 2006; Agnew, et al. 2002; Agnew \& White, 1997).

The first source of strain — the blocking of a goal or stimuli—includes the remnants of the original definition from the classic strain theories. These theories argued that strain was created from a disparity between an individual's aspirations for societal or economic success and their expectations that they will attain that success (Merton, 1938; Cohen, 1955; Cloward \& Ohlin, 1960). GST retained the frame of this definition but recognized the limitations of the previous theories by identifying that blocked goals outside of economic goals are also sources of strain (Agnew, 1992). The original source of strain was measured as the difference between a person's societal or subcultural definitions of success, such as the "American Dream" and their realistic expectations to 
attain those goals (Merton, 1938). In tweaking that definition, Agnew (1992) eliminated the structural aspect of "subcultural definitions of success," noting weak support for the idea that adolescents were driven to delinquency by their failing to meet societal aspirations like educational or occupational attainment (Agnew,1992; 2001; Agnew \& White, 1992; Paternoster \& Mazerolle, 1994). The final new strain specified under this source are strains originating from a disparity between just/fair outcomes and actual results (Agnew, 1992; 2001). However, Agnew later updated his theory, stating that injustice was not a distinct source of strain but instead was a characteristic of strain experienced across all three source categories (Agnew, 2001).

While the inspiration for the first source of strain — goal blockage — came from classic or more traditional strain theories, the remaining two sources of strain were drawn from the literature on stress and aggression (Agnew, 1992, 2001, 2006). The second source of strain refers to the loss of positive stimuli. This includes strains such as breakups, the death of a loved one, moving away from friends, or parental divorce (Agnew, 1992; Agnew, 2001). The final source of strain is created from the presence of a noxious stimuli, such as physical pain or discomfort. Again, it should be noted that while Agnew (1992) specified these sources of strains as distinct, he goes on to explain that many specific strains could be argued as coming from two or more sources (Agnew, 1992, 2001, 2006). Any of these sources could potentially result in delinquent means of coping; however, very few people who experience strain from each of these categories resort to delinquent or criminal coping (Agnew, 1992, 2001, 2006). For this reason, it is important that GST attempts to describe what characteristics of strains are most associated with delinquent outcomes. 


\section{Forms of Coping}

One major reason for these individual differences in reacting to strain is whether a person has the skills necessary to employ positive coping techniques. Individuals who are capable of employing various forms of positive cognitive, behavioral, or emotional coping techniques to deal with the negative affective state caused by strain are less likely to resort to criminal or delinquent responses. Individuals who employ negative coping mechanisms, however, may increase their likelihood of criminal or delinquent coping.

Cognitive coping, when done positively, reduces the negative emotions caused by strain through the process of reframing the problem to minimize the perceived effects, changing one's goals to alter the actual effects, or attributing the cause of the strain to themselves. For example: reassuring oneself that failing to achieve a goal does not greatly impact their overall life goals, changing one's overall life goals in the face of a major strain, and taking personal blame for the blocking of any goals are all forms of positive cognitive coping. Meanwhile, blaming others for personal shortcomings or failure or having strains that cannot be rationalized away (e.g. abuse) are negative coping techniques related to increased delinquency (Agnew, 1992).

Behavioral coping refers to non-delinquent or delinquent actions taken to reduce the strain directly through attaining a blocked goal through new means (e.g. G.E.D., sneaking out to see friends), protecting/retrieving removed stimuli (i.e. stealing back confiscated items) and removing/escaping the noxious stimuli (i.e. moving schools, running away from abusive home) (Agnew, 1992). Finally, emotional coping involves actions that attempt to directly improve/influence the negative affective state being 
experienced rather than reinterpret it as less important and includes positive coping such as exercise and meditation, and negative coping like drug and alcohol use (Agnew, 1992).

GST argues that forms of delinquent coping are likely when: 1) costs or controls on non-delinquent coping are high (i.e. low access to delinquent means), but costs or controls on delinquent coping are low (i.e. high access to delinquent means) and 2) the individual has a personal predisposition to delinquent coping (Agnew, 1992, 2001, 2006; Agnew \& White, 1992). These factors are influenced by things such as personal temperament, self-esteem, self-efficacy, problem-solving skills, social support, levels of social controls, perceptions about the cause of the strain, and the association with delinquent peers (Agnew, 1992; Agnew \& White, 1992).

\section{Types of Strain}

Before describing the types of strain that Agnew (2001) theorizes to be associated with delinquent outcomes, it is important to first explain his concepts of "objective" and "subjective" strain. Objective strains refer to a situation that the majority of a selected

group would describe as aversive (Agnew, 2001). For example, most people would agree that criminal victimization, divorce (whether from the perspective of a child or an adult) and struggling to afford basic necessities are negative situations and, therefore, objective strains (Agnew, 2001). While most people experience objective strain and cope in a nondelinquent or non-criminal manner, GST proposes that it is the individual's personal assessment of the objective strain that influences whether crime and delinquency are a likely result (Agnew, 2001).

Borrowing from the stress literature, subjective strains refer to strain disliked by the individual experiencing the strain and understanding the individual differences in 
responses to objective strain is important for GST's viability as a criminological theory. Two people may dislike an objective strain with different levels of intensity, meaning they are experiencing different levels of subjective strain. For example, a student who consistently earns low grades is less likely to report a strong emotional reaction to failing a test compared to a student with consistently high grades (Agnew, 1992, 2001). Both students experience the same objective strain (i.e. the failing test grade). However, due to the differing levels of subjective strain experienced (i.e. the strength of their reaction) and personal variables (i.e. expectations from previous tests), the students would react differently. As Student A was used to failing, their subjective reaction to the strain will be weaker than that of Student B, to whom this experience is novel. This emotional state would then make Student B more likely to respond with delinquent behavior (i.e. lying to their parents about their grade) in order to alleviate effects of the strain.

Agnew (2001) specifies that subjective strain is closely related to the negative emotional reaction an individual has to an objective strain, but that the two should be considered distinct variables, specifically because subjective strain does not include the physiological changes required in the full definition of emotional responses. He continues by saying that some people may also exhibit differences in how they interpret the same level of subjective strain. For example, two people may experience the same level of subjective strain- both dislike a certain condition equally- yet, one may react with anger while the other reacts with depression. Individuals may also vary in the intensity of their emotional reaction to identical reports of subjective strains. That is, one individual could experience mild anger while the other experiences intense anger. The types of 
objective/subjective strains experienced, as well as, other personal and social factors, influence an individual's emotional reaction to subjective strain (Agnew, 2001).

To summarize, objective strain is the precipitating event, which an individual assesses, based on personal characteristics such as access to personal and social coping skills and resources, and other life circumstances. This personal assessment is the individual's level of subjective strain, which influences emotional responses and their likelihood of reacting with criminal or delinquent coping. According to Agnew (2001), subjective strain could help to explain why some individuals react to strains through crime/delinquency while most do not (Agnew, 1992; Agnew \& White, 1992). Both objective and subjective types of strain are closely linked to the emotional and delinquent reactions to strain, but only serve as two influencing factors of many.

\section{Characteristics of Strains related to Delinquency}

Everyone experiences strain and negative emotion, but not everyone responds to strain with criminal/delinquent coping. One of GST's biggest concerns, then, is locating the reasons for individual differences in criminal/delinquent coping. Agnew (1992, 2001) argues that strains with specific characteristics are more highly associated with criminal/delinquent coping compared to others. Below, the characteristics of strains that Agnew (2001) proposes are most likely to lead to criminal/delinquent coping are discussed.

The first characteristic of a strain more likely to be related to delinquency is whether it is (or is perceived as) being unjust. GST states that individuals expect unwritten rules like "fairness" or "equity" to dictate life events. Strain, then, can occur when the outcome of an event is out of line with an individual's expected outcome based 
on these rules (Agnew, 1992). For example: Two young people work at the same places. Person A reasonably expects to be paid the same as Person B for the same work. Even if their pay is low, as long as it is even, neither would have reason to be upset. However, if one person discovers the other is making more money for the same work, this would violate their previously held belief in a "fair" or "just" world, creating strain and potentially leading to delinquent coping (Agnew, 1992).

Although originally classified as a distinct source of strain (i.e. disparity between fair and actual outcomes), Agnew (2001) later changed his definition to say that strains from any source can be seen as varying degrees of unjust. He argued that because perceived injustice is related to higher levels of anger in individuals and anger is the emotion most associated with rates of crime and delinquency, then strains perceived as more unjust should be more strongly associated with delinquency (Agnew, 2001). According to Agnew (2001), anger blocks cognitive processes needed for positive coping mechanisms, causing people to ignore other resolutions to the strain, creating an internal sense of power and control. When this feeling occurs with the subjective strain of injustice, it is possible that the individual may believe that they are "correcting" an unfair situation, absolving any guilt they would feel about criminal/delinquent coping or diminishing any controls that may have been holding them back.

Personality traits (e.g. trait negative emotionality and low-constraint, attributional style) and access to cognitive coping skills (e.g. ability to minimize problems, change frame of mind to improve perceived outcomes) play some role in whether a strain will be perceived as unjust. According to Agnew (2001), there are several other relevant factors that indicate which types of strains are more likely to be perceived as unjust and therefore 
related to delinquency. Strains perceived as voluntarily inflicted by another person/group with the intention to cause harm are likely to be considered unjust in their application, and lead to delinquent coping (Agnew, 2001). Other factors predicting whether a strain is perceived as unjust include whether the individual believes: 1) the strain is undeserved; 2) the strain does not serve any higher purpose (e.g. religion, society, etc.); 3) the strain will cause a large amount of harm to them; 4) the process used to decide whether to inflict the strain was unfair (e.g. no voice in the decision, do not recognize/trust authority inflicting strain, no rationale provided, etc.); 5) the strain involves disrespectful, inconsiderate or aggressive treatment; and 6) The strain violates social norms (Agnew, 2001).

The second characteristic of strain likely to lead to delinquent coping is the perceived magnitude. This refers to a combination of measures like the degree, duration/frequency, recency, and centrality of the strain which, when taken together, provide an idea of the negative impact of a given strain (Agnew, 2001). Degree is the term used to attempt to describe the "size" of the strain, which can be measured by either a standard metric (e.g. total amount of money lost) or in more abstract ways (i.e. intensity of negative emotions created). Regardless of the measure used, the higher the reported degree, the higher the magnitude is perceived (Agnew, 2001). Duration/frequency refers to how long an individual has experienced a strain, or how often a straining event/situation occurs. Agnew (1992) argues that some individuals who experience chronic strains (long duration) or high frequency strains may be more related to criminal/delinquent coping because the exposure to constant strain creates a 
predisposition for negative emotions. Chronic and high frequency strains, then, are also indicative of high magnitude strains.

Recency, simply put, indicates how long ago the strain occurred; with more recent strains having more of an impact on criminal or delinquent coping Although strains that have occurred more recently are seen as being higher in magnitude, Agnew recognizes that some strains that occur during formative years of childhood may be high in magnitude, despite their low recency. The last component of magnitude is centrality, which takes into account subjective opinions of the strain.

Centrality refers to how important the strain is to an individual's life goals, personal values, or identities (Agnew, 1992, 2001). Strains that interfere with these goals, values or identities are said to be higher in magnitude. For example, while a student with no college ambitions may not find poor grades to be a strain high in magnitude, one with aspirations for a full-ride scholarship would see those poor grades as far worse. Therefore, strains that have a high measure of degree, are of chronic duration or high frequency, occurred (relatively) recently, and negatively impact one's life goals, values, and identity are said to be high in magnitude. The more of these conditions that are met, the higher the magnitude is considered, and the more likely the strain is associated with criminal or delinquent coping (Agnew, 1992, 2001).

The third characteristic associated with delinquency are strains associated with or caused by low social control. Strains related to a lack of direct control (e.g. erratic discipline by authority figures), low commitment to conventional social goals (e.g. working in secondary labor markets) and low attachment to conventional others (e.g. parental neglect, negative relationships with peers) are proposed as more likely to lead to 
delinquent coping (Agnew, 1992, 2001). Strains that are associated with low social control reduce the costs of crime and/or indicate limitations on the personal resources and social support needed for non-delinquent coping. Agnew (2001) also specifies that while there can be strains experienced by individuals with high measures of direct control, attachment, and commitment, those strains are unlikely to result in delinquent coping because they are formed by high levels of social control.

The final characteristic of a strain likely to result in delinquency is whether the strain itself creates an incentive for delinquent coping. This characteristic looks at strains, which involve the modeling or reinforcing of delinquent behavior or providing definitions favorable to crime (e.g. substance abuse, peer bullying) (Agnew, 2001). Other ways strain can provide pressure for delinquent coping is when the only effective solutions in combating an unjust or high magnitude strain is through illegitimate means. For example, peer bullying is a situation in which the bully victim does not always have the option to move to a different school or class. Parents, teachers, and peers may not react to complaints of the bullying or may provide inadequate solutions that can make the situation worse. In some cases, the bullying may be so severe (i.e. high magnitude) that it might block the ability to reduce the negative emotions of the strain through other nondelinquent coping methods (Agnew, 2001). When all of this happens, delinquent coping like fighting or skipping school may occur because they are seen as the most effective solution in escaping or reducing the strain.

Drawing on these four characteristics of strains, Agnew (2001) listed some specific types of strain that he theorized would be likely to result in higher chances of criminal/delinquent coping. These specific strains include strains such as parental 
rejection, child abuse, erratic/excessive parental discipline, criminal victimization, failing a core life goal that is easily achieved through crime, and experiences with prejudice or discrimination as those likely to have strong associations wit/77];[k7\];k/]7]III/7][h delinquent coping (Agnew, 2001). On the other hand, strains lacking these characteristics, like those strains perceived as deserved (i.e. "just") or that are low in magnitude, were thought to be either unrelated or to have weaker associations with delinquent coping. Examples of these strains include unpopularity with peers (especially with delinquent peers), any strains stemming from the care of a child/sick loved one, consistent and fair parental discipline, and the failure of a goal unattainable through criminal means (Agnew, 2001).

\section{Negative affect's mediating effect on strain}

GST relies on factors such as personal resources (e.g. money, self-esteem, selfefficacy), social support, social control, coping skills, and access to delinquent peers/means in order to explain individual differences in criminal/delinquent coping (Agnew, 1992, 2001; et al., 2002). These factors are important in determining how an individual perceives the costs of criminal/delinquent coping and the differences in their predispositions towards criminal/delinquent coping methods. According to GST, however, the most important factor in why some individuals respond to strain with criminal/delinquent behavior remains the negative affective state created from an individual's level of subjective strain (Agnew, 1992, 2001, 2006; Agnew et al. 2002). Without a state of anger, anxiety, depression, or frustration, there is nothing creating a pressure to cope, which could ultimately lead to criminal or delinquent coping. 
Agnew (2006) summarized that, in addition to creating the corrective pressure, negative emotions can reduce one's ability to use non-delinquent coping, reduce care over the costs of delinquent coping, and increase one's disposition for delinquent coping. He pointed to anger's blocking effects on positive coping (i.e. narrow-mindedness, feeling justified due to perceived unjust outcomes, etc.), reduction of the perceived costs (i.e. creating a sense that any action taken is "just") and links to aggressive behavior as an example. While anger is potentially the most important emotion in relation to mediating crime and delinquency (Agnew, 2001, 2006; et al. 2002), GST specifies that any negative emotional state (e.g. depression, anxiety, frustration, fear, etc.) could be the mediating factor between strain and crime/delinquency, with some emotions being more related to some types of crime/delinquency than others. For example, jealousy might be more related to vandalism while anger is more related to physical assault (Agnew, 2006).

Agnew (2006) specifies the need to differentiate emotional states and emotional traits in regards to their relation to GST. Emotional states refer to an active instance of experienced emotion and is what GST originally meant when it referred to emotion as having a mediating effect on strain and delinquency. Emotional traits, however, refer to an individual's predisposition to respond to experiences with particular emotional state more frequently (Agnew, 2006). Trait emotionality is important to consider due to its effects on the individual's perception of strain and coping response, which raises the question of how people develop these traits.

One factor discussed as contributing to the development of emotional traits could be the draining effect of chronic or high frequency strains, which could exhaust an individual's personal resources and access to positive coping skills. This might make 
new, additional strains elicit a stronger, negative emotional reaction more often due to drained emotional coping skills, resulting in a higher likelihood for criminal/delinquent coping (Agnew, 2006). For example, an individual under the chronic strain of poverty might perceive their financial situation as unjust and develop trait anger over time, causing them to be more likely to respond to aversive situations with state anger, in turn blocking positive coping mechanisms and resulting in a higher chance for criminal/delinquent coping (Agnew, 2001, 2006; et al., 2002).

Agnew et al. (2002) investigated another factor that effects negative emotional traits: personality. While some emotional traits develop and change over a person's lifetime, Agnew and his colleagues theorized that some people may have permanent, negative personality traits that contribute to increased negative emotional reactions. Coming from the psychology literature, the "Big 3" master traits consist of positive and negative emotionality, and constraint. These are clusters of smaller, highly correlated personality traits, which are believed to be mostly stable across a person's lifetime (Agnew et al., 2002). Similar to specific trait emotions, positive and negative emotionality refer to clusters of personality traits that influence an individual's emotional response when experiencing an ambiguous situation (Agnew et al., 2002). Individuals with negative emotionality are more likely to regard a situation as aversive, accuse others of hostile intentions, engage in intense emotional reactions, and respond to these situations with aggressive behavior (Agnew et al., 2002). The trait of constraint is associated with individual measures of self-control (i.e. both will power and commitment to conventional norms/goals). Low levels of this trait, then, are related to higher levels 
impulsivity, sensation-seeking, risk taking, rejection of social norms, and potentially disregard for the feelings of others (Agnew et al., 2002).

\section{Empirical Status of General Strain Theory}

Since its introduction in 1992, GST has received a great deal of attention in the criminological literature, finding consistent empirical support for the majority of its central ideas. Studies have tested or applied the theory to a wide variety of specific strains, emotional reactions, controls to positive coping, and delinquent or criminal outcomes with results consistently supporting GST. Most recent studies focus on the "negative affect" part of the theory. Some studies have focused on examining what types or sources of strain are most likely to evoke specific negative emotions (Bao, Haas, \& Pi, 2007; Baron, 2004; Broidy, 2001; Cullen, Unnever, Hartman, Turner, \& Agnew, 2008; Higgins, Piquero \& Piquero, 2011; Rebellon, Piquero, Piquero, \& Sherod, 2009). While other studies have focused on exploring the mediating effects these negative emotions have on strain and delinquency (Agnew et al., 2002; Aseltine et al., 2000; Bishopp, \& Boots, 2014; Mazerolle, Piquero, \& Capowich, 2003; Ménard \& Arter, 2013; Moon, Morash, McClusky, \& Hwang, 2012; Rebellan et al., 2012). Other studies, however, have aimed at finding support for the relationship between strain and delinquency without the mediation of negative affect and have returned mixed results, especially when analyzing longitudinal data (Agnew \& White, 1992; Higgins et al., 2011; Mazerolle, 1998;

Paternoster \& Mazerolle, 1994).

Agnew \& White (1992), for example, investigated the relationships of 8 specific strains, a composite measure of general strain and their relationship with delinquency, while controlling for social control and attachment. Using data from the Rutgers Health 
\& Human Development Project, they performed cross-sectional and longitudinal analyses of $552 \mathrm{New}$ Jersey adolescents (ages at collection: 12, 15 and 18). Their cross-sectional findings provided early evidence for one of the main arguments of GST: The measures of strain including negative life events (assault, death of a friend, etc.), life hassles (disliked by classmates, not respected by adults, etc.), parental fighting, and negative relationships with adults were all positive and significantly related to delinquency (Agnew \& White, 1992). Measures for neighborhood problems and all the previously significant variables, except for negative adult relations, were also positive and significantly related to reported drug use. Additionally, the composite measure of general strain was also found to have a strong positive significant relationship with delinquency, even when controlling for social control and attachment factors.

Regarding the longitudinal analysis, they found much less support for GST, with only the composite measure of strain showing a small positive significant relationship with delinquency. The authors suggest that these weak findings may be due to their data collection having a three-year lag between sampling waves. They argued that GST proposes that strain has a "contemporaneous effect" on delinquency and would require a timelier follow-up to provide a more accurate assessment (Agnew \& White, 1992). In response, Paternoster \& Mazerolle (1994) conducted a replication study, using a data set with only a one-year lag between testing periods. Using data from 1,515 adolescents (ages 11-17) gathered in the National Youth Survey, their cross-sectional analysis found some similar results to Agnew \& White's. Negative life events and negative relationships with adults, were again found, to have significant positive effects on delinquency, with 
neighborhood problems also showing significance, although to a lesser extent (Paternoster \& Mazerolle, 1994).

Unlike the previous study, however, the longitudinal analysis found significant relationships with all of the individual strain measurements except for traditional (goalattainment) strain (Paternoster \& Mazerolle, 1994). The significance of these relationships remained after accounting for the effects of social control and differential association measures such as delinquent peers or a delinquent past, grades, family attachment and moral beliefs. The importance of these conditioning variables on positive coping was also seen in Bao and colleagues' (2007) analysis of 615 Chinese students, which found that negative relationships with adults (both parents and teachers, individually) and peers, and associating with delinquent peers were significantly related to delinquency. They also found that moral beliefs and family/school attachment were strongly and inversely related to delinquency rates. That is, students with stronger moral beliefs and family/school attachment were less likely to be involved in delinquency.

The relationship between various individual measures of the three sources of objective strain, composite measures of these strains, and measures of delinquency are supported in other tests of GST (Bao et al., 2007; Baron, 2004; Baron \& Hartnagel, 2002; Mazerolle, 1998; Higgins et al., 2011). However, by not measuring levels of subjective strain or the mediating effects of emotions on strain and delinquency, these studies fail to account for GST's distinguishing feature from other strain theories which may explain some of the mixed support found for individual strains such as neighborhood problems or traditional strain (Mazerolle, 1998; Paternoster \& Mazerolle, 1994). 


\section{Tests of Emotions as Mediators}

The main difference between traditional strain theories and GST, aside from its expanded definition of strain, is the addition of the psychological-level interest in an individual's subjective reaction to their experienced strains. The proposed mediating effect of negative affect between strain and delinquency is at the heart of Agnew's GST, and unsurprisingly, has been tested thoroughly with ample support. While much of the literature examining the mediating effect of negative affect focuses on the effects of anger across different samples (Agnew et al. 2002; Broidy, 2001; Mazerolle et al., 2003), support for the effects of depression and other negative emotions has also been found (Aseltine et al., 2000; Moon et al., 2012; Rebellan et al., 2012; Rebellon et al., 2009).

Agnew et al.'s (2002) study aimed to expand GST and further support the idea that an individual's emotional reaction was what led to their delinquent coping by examining chronic, trait negative emotionality and how it related to delinquency. Using a sample of over 1,400 school children, Agnew and his colleagues hypothesized that individuals with high scores in "Big 3" trait negative emotionality and low measure of "Big 3" trait constraint would have a strong, positive effect on delinquent coping. Their analysis confirmed their hypothesis, indicating that trait negative emotionality/low constraint were strongly related to measures of delinquency (Agnew, et al., 2002). The positive significant relationship between trait negative emotionality and delinquency implies that people who are likely to blame others for negative situations and are prone to stronger emotional reactions are more likely to employ delinquent coping techniques. These findings provide strong support for the idea that trait emotionality (whether developed or static) is related to individual differences in criminal/delinquent coping. The 
findings also support the larger central argument of GST that negative emotions act to mediate the effect of strain on delinquent behavior. While the study provides strong support for GST as a whole, it also lays a foundation in beginning to explain the different reasons some people react with delinquency and others do not.

Aseltine, Gordon, and Gore (2000) provided more support for GST with their longitudinal study of approximately 1,200 high school students where they aimed to test all of the theory's "core tenets." Their results found that anger and anxiety, on their own, did not have a direct effect on any measure of delinquency; however, there was a strong direct effect between family conflict (i.e. strain) and aggression (i.e. reported violent delinquency) that was completely mediated by anger. Aggression's relationship to negative life events was only partially mediated by anger (Aseltine et al., 2000). Other results regarding anxiety's effects on delinquency and marijuana use found that anxiety was not a mediating factor.

Anger also mediated all or most of the relationships between measures of strains perceived as unjust and delinquency, with unjust strains also having a strong positive effect on anger itself (Broidy, 2001; Rebellon et al., 2012; Rebellon et al., 2009). In an experimental test of GST, Rebellon et al. (2009) exposed university students to the same scenario, which led to one of three different endings: the "expected" outcome, an unexpected but fair outcome, and an unfair outcome. The results found that the group exposed to the unfair outcome of the story reported more situational anger, which in turn, was linked to significantly higher levels of self-reported likelihood of engaging in theft. Broidy's (2001) study investigated the relationship between emotions, specifically anger and other "negative emotions" (e.g. depression, loneliness, frustration), and all 
forms of strain in a sample of over 800 college students. Consistent with other findings on strain and anger, all types of strain were significantly related to higher levels of reported anger (Rebellon et al., 2012; Rebellon et al., 2009). Another finding of note was that only one type of strain, the presentation of noxious stimuli, was found to be significantly related to negative emotions including depression (Broidy, 2001). This provides support for the idea that other negative emotions beyond anger are also caused by strain and that these emotions can mediate the relationship between strain and delinquent or criminal coping (Broidy, 2001).

Moon and colleagues (2012) used self-report data from 659 Korean schoolchildren to examine the relationship between the negative emotions of anger and depression, and violent delinquency. In an effort to test GST, the researchers created separate measures for trait-emotions and state-emotions and included these both in the analysis. Consistent with other findings, anger at the trait level was positively and significantly related to all violent delinquency measures (Mazerolle, et al. 2003; Moon et al. 2012). Trait-depression had no significant relationships with violent delinquency. Situational anger (i.e. state anger) was positively and significantly related to all but two self-reported measures of violent delinquency, whereas situational depression was inversely, significantly related to several measures of violent delinquency (Moon et al, 2012). This provides further support for the idea that different negative emotions can influence different forms of delinquency, and that trait and state emotionality influence whether an individual reacts with delinquency (Mazerolle et al. 2003; Moon et al., 2012). 


\section{Coping as a Mediator}

While some studies testing aspects of GST continue to focus on explaining which negative emotions are related to which strains or are more likely to lead to delinquency, other studies have examined why some people resort to delinquency when most people respond to strain or negative emotions in a non-delinquent way. For example, Brezina (1996), found using longitudinal data from 1,799 high school aged boys that when someone takes part in delinquent coping, it significantly reduces the negative emotion felt from the strain. Paired with other findings from Broidy's 2001 survey study that the relationship between delinquent coping and "negative emotions" (not including anger) were mediated by positive coping factors, such as social controls and attachment levels. This suggests that one of the biggest differences between those who employ delinquent coping are those who frequently experience anger or those who do not have access to social support, personal resources, and weak social controls and attachments (Brezina, 1996; Broidy, 2001). This supports other findings on coping access and delinquency that are often included in studies focused largely on negative emotions as a mediator.

\section{GST and Anti-Social Coping}

As GST is a "general" theory and, therefore, meant to explain as wide of a variety of criminal and delinquent behavior, it only makes sense that it is also applied to broader deviant coping mechanisms, that are not necessarily illegal, as well. Ménard \& Arter (2013) makes a great example of how GST's frame can be applied beyond the explanation of delinquent coping mechanisms: The strain of a traumatic workplace event, mediated by an extreme emotional reaction such as depression, can result in the deviant coping of alcohol abuse. 
Ménard \& Arter (2013) applied GST in an attempt to explain the high rates of alcohol use and PTSD among police officers. The authors explain PTSD and alcohol abuse are caused by a lack of social support and the use of negative or avoidant coping strategies. After officers were exposed to situations such as shootings, physical assault, or the aftermath of a violent accidents (i.e. "critical incidents"), they fail to properly cope and develop PTSD and/or abuse alcohol as a deviant coping technique (Ménard \& Arter, 2013). Their findings indicate that although strain measures and alcohol use were significantly related, the significant relationship is lost when positive coping skills (e.g. social support) are included in the model. This suggests that alcohol use is mainly the function of a lack of positive coping skills (Ménard \& Arter, 2013). Social stressors (e.g. feelings of public disapproval) did not have a significant relationship with alcohol use. Critical incidents, coping mechanisms, and alcohol use all displayed positive, significant relationships with higher rates of PTSD symptomology as measured by the PTSD Checklist-Civilian Version. The significance of all of these factors were mediated by coping skills and social stressors (Menard \& Arter, 2013). This suggests that while critical incidents were strongly related to higher measures of deviant coping (i.e. alcohol use) or severe negative affective states (i.e. PTSD), that these responses are significantly mediated by the access to prosocial coping mechanisms and social support systems. In short, they provided evidence that a lack of access to legitimate coping for emotional strain can lead to deviant behavior, which is GST's central argument.

There have been several studies, which have applied the effects of strain directly to suicide, both in the high-risk population of policing, as well as, the general population (Bishopp \& Boots, 2014; Zhang \& Lester, 2008). Bishopp and Brooks (2014) took a 
quantitative approach to the same question, while also considering potential gender differences in the effects of strain on suicidality among police officers. Acknowledging that more police die by suicide than by occupational homicide (Bishopp \& Brooks, 2014), the authors sought to examine the relationships between occupational stressors, gender, and suicidality. Using surveys from 1,410 police officers, the researchers examined the relationship between reported violent incident exposure and suicidal ideation, as well as, their relationships when mediated by feelings of anger, depression, and burnout.

Despite the studies focus on gender, one of the most relevant findings was that when both genders were considered, measures of depression and anger fully mediated the significance of the direct effect strain had on suicidal ideation (Bishopp \& Brooks, 2014). This is evidence that GST is likely a viable framework for suicide as a coping response to occupational stressors. Other findings from the study highlighted the differences between genders, including that strain had a significant, direct relationship with suicidal ideation in men, whereas among female officers, the direct relationship fell short of significance (Bishopp \& Brooks, 2014). The negative emotions of anger and depression fully mediated the relationships between strain and suicidal ideation in both genders; this is consistent with GST's main argument that strain's role in maladaptive coping is mediated by the negative emotions the strain has caused.

Zhang and Lester (2008) examined what they called the "Strain Theory of Suicide" by conducting a qualitative study of 40 suicide notes ( 20 completed suicides, 20 attempted). The authors coded four types of strain they believed could lead to deviant coping. These four types include strains of value, aspiration, deprivation, or coping. 
Value strain occurs when personally held values contrast with a person's everyday life. Aspiration strain occurs when a person realizes their aspirations are not in line with the reality of their life. Deprivation strain is felt when an individual compares their current circumstances to someone from a similar background who they feel is more successful. Finally, coping strain occurs when a person is ill-equipped to emotionally handle and aversive situation or stimuli.

Out of all 40 suicide notes, only one did not contain evidence of any of the four types of strain, while the majority of notes contained at least two or more types of strain; the most common being Aspiration strain $(n=38)$ and Coping Strain $(n=37)$ (Zhang and Lester, 2008). The study did not include a measure for negative affective states, which GST claims are responsible for maladaptive coping, but this study's measures of strain greatly overlap with Agnew's (1992; 2001) broad sources of strain (Zhang \& Lester, 2008). Their findings that strain was present in almost every letter, then, provides further evidence for GST as a potential model for understanding suicide. Negative emotionality caused by a perceived inequality or injustice, combined with a lack of access to positive coping methods, can lead to the adoption of deviant coping techniques, in order to alleviate the strain. Unfortunately, in this case, that coping mechanism is the most permanent and negative--suicide.

\section{The Current Study}

With this existing research in mind, the current study utilizes a GST framework for examining the relationship between PTSD, depression, and suicidality. As seen in the existing literature, there is a connection between measures of strain as the presentation of a noxious stimuli and PTSD, including critical events (i.e. those police officers 
experience in the line of duty) (Ménard \& Arter, 2013). The theory also indicates that actions done voluntarily and with the intention to cause harm (e.g. assault or criminal victimization) increase trait and situational anger, which are both related to aggressive and violent criminal/delinquent coping (Aseltine et al., 2000; Cullen et al., 2008; Mazerolle et al., 2003; Moon et al., 2012; Rebellan et al., 2012; Rebellon et al., 2009). This suggests that traumatic events that can increase the chance that PTSD may lead to aggressive criminal or delinquent coping. PTSD, depression, and suicidal ideation are all also more common in those with poor coping skills and limited social support (Agnew 2001; Agnew et al., 2002; Bishopp \& Brooks, 2014; Ménard \& Arter, 2013). Considering that those with limited access to proper coping techniques already seek criminal or delinquent means of coping, it would follow that individuals with PTSD might react with higher rates of suicidality, especially when it is mediated by the negative state of depression.

As PTSD can only occur in the aftermath of a traumatic experience, it can be used as an analog measure for strain. Specifically, it could fall under all of Agnew's (1992) three broad categories of strain: 1) PTSD prevents the individual from achieving the positively valued goal of forgetting/moving past their trauma through flashbacks and intrusive episodes; 2) It's creation of avoidant behaviors could cause the removal of positively valued stimuli (e.g. restriction from previously enjoyed activities or selfseclusion to avoid triggers); and 3) both the initial trauma and the newly acquired (and now recurring) symptoms of PTSD can be considered a presentation of "noxious" or negative stimuli (Agnew, 1992; 2001; Agnew et al., 2002; APA, 2013). GST mentions 
depression as a specific measure likely to mediate strain's relationship with crime and delinquency.

While support for depression as a mediator for violent delinquency is inconsistent across general forms of delinquency in the general population (Broidy, 2001; Moon et al., 2012), there is strong evidence that depression has a mediating effect on suicidal behavior (Bishop \& Boots, 2014; McKinney et al., 2017). This would support exploring whether depression mediates PTSD's effect on deviant coping, such as suicidality. There is powerful support for the relationship between strain and suicide, especially strains that are accompanied by a lack of proper coping strategies (Bishop \& Boots, 2014; Zhang \& Lester, 2008). So, while suicidal thoughts and behaviors are not necessarily criminal or delinquent coping, they can still be examined through a GST lens as antisocial coping mechanisms.

Correctional staff, who also experience critical incidents similar to police officers, report high levels of occupational violence, PTSD, and depression (Denhof \& Spinaris, 2013, 2016; Spinaris, Denhof \& Kellaway, 2012, Swartz et al., 2017), gain a great deal depending on the results of the study. For these reasons, the current study proposes that the issue of suicidality among correctional staff be viewed as a byproduct of strain. That is, the development of PTSD following a critical incident feeds an additional negative affective state of depression, and without access to proper coping, correctional staff turn to suicidality as their escape from the strain. 


\section{CHAPTER 4: METHODS}

The previous sections have demonstrated that using GST as a potential framework for examining the relationships between PTSD, depression, and suicidality is feasible. This framework implies that strain leads to a negative affective response, and that to alleviate this negative emotional state, an individual might resort to antisocial coping. The current study applies this framework to the problem of PTSD, depression and suicidality by examining whether the effects of PTSD (acting as the strain) on suicidal ideation are mediated by the development of depressive symptoms. The following section describes the sample, measures, and analysis used in examining these relationships.

\section{Sample}

This study performed secondary analysis on cross-sectional data originally collected by a team led by Dr. Kristin Swartz, of the University of Louisville, working in collaboration with the Kentucky Department of Corrections (KYDOC). Noting a trend of research indicating that correctional staff reported significantly higher rates of PTSD than other high-risk occupations (Denhof \& Spinaris 2013, 2016; Spinaris, Denhof, \& Kellaway, 2012), KYDOC reached out to Dr. Swartz about conducting a further study. The data collection period occurred from May to December of 2015 and included staff 
from all 12 adult correctional facilities in Kentucky. The overall KYDOC staff population is just over 3,500 (Commonwealth of Kentucky, 2017) and the goal was to survey $30 \%$ of all institutional correctional staff, which would have been 800 staff. A total of 803 staff completed the surveys, but after considering missing data, only 775 participants are included in the analyses here. The sample was representative of the overall KYDOC correctional staff population. The average age of the sample was 39.5 years (SD: 11.4 ) and the average age of KYDOC staff is 40 years. The sample was $63.9 \%$ male and $36.1 \%$ was female, with no respondents identifying as transgender. This is representative of the overall KYDOC staff population with $36 \%$ of staff being female. Race was collapsed to a dichotomous measure to mirror the racial classifications reported by KYDOC. The sample was $92 \%$ white and $8 \%$ nonwhite. This too is representative of the KYDOC staff population, which is $92.4 \%$ white and $7.6 \%$ nonwhite. The reported level of education attained by respondents was collected by asking to signify the highest level of education attained by marking a 1 (some high school) through 6 (graduate degree): The average participant responded with a 3.17, or that they had "some college" (SD: 1.14). $33.8 \%$ of the respondents reported completing high school, $30 \%$ reported some college, $26.5 \%$ had a college degree, $6.7 \%$ had a graduate degree, and $2.6 \%$ had some graduate school. Most staff reported working in a security or custody position with $59.5 \%$ of working as security staff and $40.5 \%$ working in a non-security position.

Participants responded to a variety of self-report surveys and assessments including demographics (age, race, gender, education, military history, etc.), life-stress events, job burnout and satisfaction, personality, attitudes towards both coworkers and the prison environment, violent/traumatic event exposure, and an assessment of mental 
health symptomology. The focus of the current project is on the relationships between the symptoms of these various mental health issues, more specifically, the relationships between PTSD, depression, and suicidality. These symptoms were measured using the Trauma Symptom Inventory-2 (TSI-2) (Briere, 2011), a self-report questionnaire containing 12 scales measuring the various types of trauma-related symptoms behavior that can develop following traumatic event.

\section{Trauma Symptom Inventory-2}

Revised from Briere's (1995) Trauma Symptom Inventory, the TSI-2 is a 136 item self-report questionnaire designed to measure a variety of short and long-term symptoms developed after a traumatic event (Briere, 2011). Each TSI-2 item asks participants how frequently they experienced a specific trauma-related symptom or behavior (e.g. "Feeling jumpy"; “Avoiding relationships with people”) on a scale of 0 (Not at all) to 3 (Often), within the last 6 months (Briere, 2011). Each item is assigned to one of the 12 clinical scales measuring a specific trauma-relate symptom or behavior (e.g. depression, anxiety, suicidality, etc.) or one of the 2 validity scales designed to detect false positives and other irregular response patterns. These 12 clinical scales allow researchers to examine the various complex symptom responses that can be exhibited depending on the sample and trauma experienced (Briere, 2011). It is important to note that despite its ability to measure a wide range of symptoms related to mental disorders, the TSI-2 is not a diagnostic tool for any disorder whose symptoms it measures. Instead, the TSI-2 allows clinicians and researchers to determine whether the symptom levels reported by individuals or within a sample were elevated to a "problematic" or "clinically elevated" degree, relative to a normalized sample (Briere, 2011). In other words, the TSI- 
2's clinical scales display which symptoms (if any) respondents are reporting at significantly higher rates than a randomly selected sample.

Briere (2011) sorted the 12 clinical scales into four factors: Self-Disturbance (SELF), Posttraumatic Stress (TRAUMA), Externalization (EXT), and Somatization (SOMA). The Self-Disturbance factor contains scales that measure negative changes in how respondents perceive themselves, and/or how they think they are perceived by others, following a traumatic event (Briere, 2011). This includes the Depression (D) scale, which measures Depression symptoms using that includes items such as "feeling hopeless," "low self-esteem". The Insecure Attachment (IA) scale, which measures problems with interpersonal relationships with its subscales: The IA-Relational Avoidance (IA-RA) subscale (e.g. "not letting people get to know you very well") IARejection Sensitivity subscale (e.g. “worrying someone didn’t like you anymore”). And finally, the Impaired Self-Reference (ISR) scale measures changes to negative selfperception using two subscales: The ISR-Reduced Self-Awareness (ISR-RSA) subscale (e.g. "feeling like you don't know who you are") and the ISR-Other-Directedness (ISROD) subscale (e.g. "your opinions change depending on who you are with") (Briere, 2011). The scales and subscales making up the Self-Disturbance factor and all other TSI2 factors can be seen in Figure 1 below. 
(Figure 1: A model depicting the TSI-2's four factors and their scales and subscales)
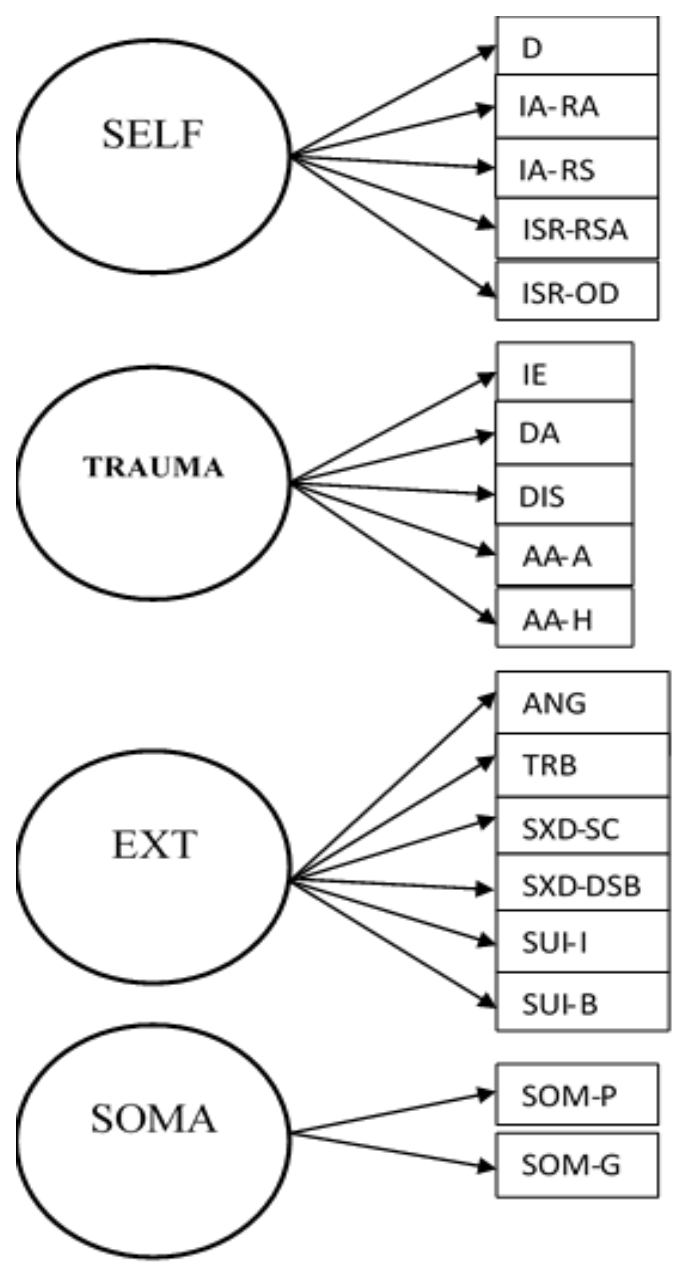

The TRAUMA factor is made up of all the scales that represent the DSM-IV symptom clusters of PTSD: The Intrusive Experiences scale (IE), the Defensive Avoidance Scale (DA), the Dissociation scale (DIS) and the Anxious Arousal scale which is separated into Anxiety (AA-A) and Hyperarousal (AA-H) subscales (Briere, 2011). Again, it should be noted that the TSI-2 is not meant to be diagnostic tool for PTSD or any disorder include within the clinical scales. Instead, "clinically-elevated" scores across the scales of the TRAUMA factor indicate a trauma symptomology suggestive of a likely PTSD diagnosis if given a clinical exam (Briere, 2011). Within the 
current study, this was the only factor to retain all of its scales, the details are discussed later in this section.

The Externalization factor is comprised of all the scales that represent the different ways an individual might "act out" due to their disruptive emotional state following a traumatic event (Briere, 2011). This factor consists of the Anger (ANG) scale (e.g. "yelling or telling people off," "thoughts or fantasies about hurting someone"), the Tension Reduction Behavior (TRB) scale (e.g. "doing something violent because you were so upset," "punishing yourself so you would feel less guilty"), and the Suicidality (SUI) scale, with its Suicidal Ideation (SUI-I) and Suicidal Behavior (SUI-B) subscales (e.g. "suicidal thoughts" and "trying to end your own life") (Briere, 2011). Finally, the Somatization factor consists of reports of physical symptoms following trauma. This factor uses only the Somatic Preoccupations (SOM) scale, measuring reports of symptoms such as "aches and pains," "muscles spasms," "nausea or an upset stomach," and "ringing in your ears" with its two subscales: SOM-Pain (SOM-P) and SOM-General (SOM-G) (Briere, 2011).

To determine a respondent's score (i.e. level of symptom elevation) all of the 136 items are first separated into their related scales, then the item scores of each scale are totaled to provide the scales" "raw score." These scores can then either be compared to the scores of a normalized sample in order to determine significance or summed into their larger factors. However, for the purposes of the current study, the raw score and significance were not calculated for any scale or factor. The specific type of analysis used is discussed in the following section. 


\section{Analysis and Variables: Structural Equation Modeling}

This study used structural equation modeling (SEM) via MPLUS (3.11) for its analysis. SEM is an analytic process that combines aspects of several other techniques (e.g. Confirmatory Factor Analysis, Multiple Linear Regression, and Path Analysis) to attempt to find the best explanation for covariance within a sample (Higgins, Fell, \& Wilson, 2006; Hooper, Coughlan, \& Mullen, 2008). Conducting SEM, then, requires that there be multiple, significant bivariate relationships (i.e. covariance). This allows for multiple explanations (i.e. structural path models), which are often guided by preexisting theory, to be tested and compared against each other to determine which model best explains the variation among the sample.

\section{Independent Variable- Posttraumatic Stress Disorder}

The latent variable used to represent PTSD in the analysis was constructed from the 4 TRAUMA factor scales (i.e. Intrusive Experiences, Defensive Avoidance, and Anxious Arousal). As a valid measure of PTSD was central to the study, it was ensured that the validity of the TRAUMA factor, initially tested by Briere (2011), was kept intact by assuring all the items within each scale of the factor were retained. It is also important to mention that, although the TSI-2 (Briere, 2011) was published based on diagnostic criteria of PTSD before the release of the DSM-V, the scales are still seen as accurately measuring all of the new DSM-V symptom clusters. The latent variable of PTSD, then, was comprised of the mean raw score of the subscales forming the TRAUMA factor, whose measures directly examine respondents' levels of reported PTSD symptomology. A more detailed description of each TSI-2 TRAUMA subfactor scale, their items, and 
their corresponding DSM-IV and DSM-V symptoms are discussed in the following sections.

\section{Intrusive Experiences}

The Intrusive Experiences (IE) scale directly examines both the DSM-IV and DSM-V "B” cluster of PTSD symptoms (APA, 2013). This group of symptoms includes involuntary and disturbing thoughts and memories, nightmares or violent dreams, and dissociative episodes (i.e. flashbacks) related to a trauma, which cause psychological distress and/or noticeable physiological reactions (APA, 2013; Briere, 2011). The TSI-2's IE scale includes 10 items, with at least one item directly mirroring each of the group "B" symptoms, for example: "memories of the past that won't go away," "flashbacks (sudden memories or images of upsetting things)," "nightmares or bad dreams," "your heart going suddenly going fast when you were reminded of something bad," and "getting upset when you were reminded of something from your past" (Briere, 2011). The next paragraph discusses the Defensive Avoidance scale, which measures a second central symptom of PTSD closely related to intrusive experiences.

\section{Defensive Avoidance}

The second TRAUMA factor scale used in the creation the latent variable of PTSD, the Defensive Avoidance (DA) scale, has 10 items that can be directly connected one of the two symptoms in the DSM-V's "C" cluster. This symptom group includes the existence of (1) repeated attempts (successful or otherwise) to avoid thoughts, memories or flashbacks related to a traumatic event and (2) attempts to avoid any external stimuli (i.e. people, places, topics, or activities) that could act as a reminder of that trauma and incite intrusive thoughts or memories (APA, 2013). The DA scale items measuring these 
symptoms include "trying to forget a bad time in their life," "staying away from certain people or places that remind you of something," "trying not to have any feelings about something that hurt you," and "pushing painful memories out of your mind" (Briere, 2011). Although the previous scales transition between editions of the DSM easily, it is at symptom cluster "C" that differences start to become apparent. Namely, the major change between the DSM-IV and DSM-V "C" symptom cluster was the moving of several symptoms related to "negative changes in cognition and mood following a trauma" into a cluster of their own, a new "D" group of symptoms (APA, 2013). The next paragraph is a discussion of the TSI-2 scales and items that manage to represent this new symptom cluster in the latent variable.

\section{Dissociation}

The third TRAUMA factor scale included in the formation of latent PTSD is the Dissociation scale (DIS) and includes 10 items that directly measure a few key symptoms from both the DSM-V's "D" and "E" symptom clusters. More specifically, it has items that directly measure the cluster "D" symptoms of "dissociate amnesia" (e.g. "having trouble remembering the details about something bad that happened to you) and "feelings of detachment or estrangement from others" (APA, 2013) (e.g. "feeling like you were in a dream," "not feeling like your real self") (Briere, 2011). The scale also contains items that directly measure the single cluster "E" symptom of "problems with concentrating" (e.g. "spacing out," "people saying you don't pay enough attention to what going on around you) (APA, 2013; Briere, 2011). This symptom is also measured on the Anxious Arousal scale, with the remainder of the "E" cluster symptoms, as discussed below. 
The final scale from the TRAUMA factor used in constructing the latent PTSD variable was the Anxious Arousal scale. This scale provides at least one direct measure for several symptoms from the DSM-V's " $D$ " cluster and five of the six symptoms from cluster "E" that were not included in the Dissociation scale. Unlike the other 3 TRAUMA scales, the Anxious Arousal scale is divided into 2 subscales: AA-Anxiety and AAHyperarousal. This provides researchers with separate measures of anxiety's cognitive and physiological hyperarousal symptoms. Due to evidence suggesting that hyperarousal symptoms show a stronger relationship to suicidality when in the presence of depression than non-physiological anxiety symptoms in military samples (Hellmuth et al., 2012), the two subscales were considered independently from one another in forming the latent PTSD variable.

\section{AA-Anxiety}

The Anxious Arousal-Anxiety (AA-A) subscale contains items that measure several symptoms from the DSM-V's “D” group. For example, “persistent negative emotional state" could be said to be measured by the subscale item asking the respondent how often they would report feeling "nervousness" or "feeling afraid you might die or be injured" (APA, 2013; Briere, 2011). For the "D” cluster symptom "persistent negative beliefs or expectations about oneself, others and the world," there are the AA-A subscale items "feeling afraid of certain things, even when there probably wasn't any real danger," "worrying about things more than you needed to," and "worrying about things more than you needed to" (APA, 2013; Briere, 2011).

\section{AA-Hyperarousal}


The items from the Anxious Arousal-Hyperarousal (AA-H) subscale measure the symptoms of heightened physiological arousal that might develop following exposure to a traumatic event. More specifically, this subscale's 5 items measure five of the six symptoms included in the "E" symptom cluster of PTSD. The symptoms measured included sleep disturbance (i.e. "trouble getting to sleep or staying in asleep because you were feeling tense"), hypervigilance (i.e. "watching out for danger), concentration problems (i.e. "having trouble paying attention because you were so tense"), increased irritability (i.e. "after a bad thing happened, feeling irritable or easily angered,") and heightened startle response (i.e. "feeling jumpy") (APA, 2013; Briere, 2011). In other words, the AA-Hyperarousal scale has an item measuring every symptom in cluster "E" except for "reckless and self-destructive behavior" (APA, 2013). Again, the subscales were considered as independent observed measures when forming the latent PTSD variable due to empirical evidence suggesting that AA-Hyperarousal (or the physiological, "E” cluster of symptoms) displays a relationship with suicidality that is distinct from that of AA-Anxiety (Hellmuth et al., 2012). Overall, the latent variable of PTSD was constructed from the mean raw scores of the TRAUMA scale, which includes 3 scales (i.e. Intrusive Experiences, Defensive Avoidance, and Dissociation) and 2 subscales (i.e. AA-A and AA-H) designed to measure nearly all of the various symptoms of the disorder.

\section{Mediating Variable- Depression}

Following the creation of the latent PTSD variable, a latent construct was formed to represent the mediating variable of depression. Items from the TSI-2's Depression scale were used to create the latent depression variable. These items directly measure 
various DSM-V depression symptoms: depressed mood ("sadness," "feeling depressed"), low self-esteem (e.g. "feeling worthless," feeling hopeless"), changes in appetite ("being so depressed you didn’t feel like eating"), feelings of anhedonia ("not enjoying things that other people enjoy because you were so depressed") (APA, 2013; Briere, 2011). The items used in the scale represent almost all of the symptoms listed as requirements for a diagnosis of depression in the DSM-V, making the TSI-2's Depression scale a reasonable measure for potential depression levels when all ten items are included.

However, due to issues with multicollinearity among the TSI-2 items used, the MPLUS software used to perform the SEM analysis rejected many of the Depression scale items used in the CFA. After reviewing the scale's items, those believed to not affect the scale's validity were removed. Items that were deemed too repetitive (i.e. "low self-esteem," "feeling bad about yourself," "hating yourself") or not core to the diagnosis (i.e. "loss of appetite from depressed emotion") were removed and the analysis was attempted again with 6 items remaining. Despite the removal of these four items, the multicollinearity between all items persisted and the MPLUS program continued to reject the remaining six items from use in the model. The same pattern was followed on the remaining Depression scale items until the software allowed for the creation of latent depression. Unfortunately, this meant the removal of seven of the ten depression scale items, including the items measuring the two symptoms of depression required: persistent depressed feelings (i.e. "feeling depressed") and anhedonia (i.e. "not enjoying activities that other people usually enjoy") (Briere, 2011). Considering that this latent variable was constructed using only three of the original ten items, and that those items remaining measured various, non-central depression symptoms, those being "sadness," 
(D-1) "being so depressed you avoided other people," (D-4) and "feeling worthless" (D5) rather than the core symptoms of the disorder, the current study is not able to claim that it measures depression, but instead examines the mediating effects of "depressive symptoms" that develop alongside the symptoms of PTSD.

\section{Dependent Variable- Suicidal Ideation}

The final latent variable constructed before the CFA was the dependent variable Suicidal Ideation. As the current study's focus is on whether depressive symptoms formed by PTSD had mediating effects on the antisocial coping mechanism of suicide, the TSI-2's Suicidal Ideation subscale was used as a measure for increased suicide risk. Although the TSI-2 includes a full Suicidality scale, the problems with multicollinearity experienced within the Depression scale occurred again with this scale. Four items of the Suicidal Ideation scale remained to be used in the final analysis. This meant that the latent dependent variable for Suicidal Ideation consisted of the TSI-2 Suicidal Ideation items: "wishing you were dead" (SI-1), "fantasies about dying" (SI-2), and "feeling so hopeless you wanted to die" (SI-3) and "suicidal thoughts" (SI-4) (Briere, 2011, p. 17).

The follow is a table including the descriptive statistics of the demographics and responses to the items used in forming the latent variables.

(Figure 2: Descriptive Statistics Table)

\begin{tabular}{lccccc}
\hline \multicolumn{1}{c}{ Descriptive Statistics } & Mean & SD & Min & Max & N \\
Age & 39.519 & 11.363 & 21 & 73 & 772 \\
Gender & 0.639 & 0.481 & 0 & 1 & 775 \\
Race & 0.924 & 0.266 & 0 & 1 & 773 \\
Education Level & 3.172 & 1.139 & 1 & 6 & 775 \\
Security/Non-Security & 0.629 & 0.483 & 0 & 1 & 773 \\
TRAUMA Factor & 38.748 & 26.142 & 0 & 117 & 771 \\
$\quad$ Anxious Arousal & 13.177 & 7.273 & 0 & 39 & 773
\end{tabular}




\begin{tabular}{lccccc} 
Intrusive Experiences & 9.000 & 8.152 & 0 & 53 & 772 \\
Defensive Avoidance & 10.690 & 8.046 & 0 & 30 & 774 \\
Dissociation & 5.885 & 5.593 & 0 & 29 & 774 \\
Depressive Symptoms & 3.340 & 2.606 & 0 & 9 & 774 \\
Sadness & 1.352 & 0.947 & 0 & 3 & 775 \\
Feeling worthless & 0.991 & 1.053 & 0 & 3 & 775 \\
Feeling so depressed that you avoided people & 0.996 & 1.093 & 0 & 3 & 774 \\
Suicidal Ideation & 1.353 & 2.564 & 0 & 12 & 768 \\
Wishing you were dead & 0.359 & 0.748 & 0 & 3 & 774 \\
Fantasies about dying & 0.367 & 0.768 & 0 & 3 & 774 \\
Feeling so hopeless that you wanted to die & 0.387 & 0.791 & 0 & 3 & 775 \\
Thinking about killing yourself & 0.233 & 0.636 & 0 & 3 & 770 \\
\hline
\end{tabular}

Once all three of the latent variables representing PTSD, Depressive Symptoms, and Suicidal Ideation were created, the analysis of their relationships through Structural Equation Modeling could continue. To examine these relationships, four different structural models are created for analysis; the following section discusses these models and their results. 


\section{CHAPTER 5: RESULTS}

Using a GST framework, the current study examined the relationships between PTSD, depressive symptoms, and suicidal ideation. GST states that a strain is most likely to result in antisocial coping when it creates a negative affective state in an individual. Using this framework, this study examined whether the direct effect of PTSD (i.e. the strain) on suicidal ideation (i.e. the anti-social coping) was mediated by the development of depressive symptoms (i.e. the negative affective state).

This study used structural equation modeling (SEM) via MPLUS (3.11) for the analyses. SEM is an analytical process that uses existing theories to attempt to explain the covariance among unobserved variables within a sample, meaning SEM requires significant covariance to operate (Higgins et al., 2006; Hooper et al., 2008; Schreiber, et al., 2006). The current study found significant bivariate relationships between a majority of both the observed and latent variables. As seen in the table below (Figure 3) one subject of note is the high levels of multicollinearity observed between all of the TSI-2 items, which are all seen as significantly correlated with one another. Beyond this, the findings that the latent variables were all found to be significantly related suggests there was enough covariance found within the sample to support further analysis (Higgins et al., 2006; Hooper et al., 2008; Schreiber, et al., 2006). 


\begin{tabular}{|c|c|c|c|c|c|c|c|c|c|c|}
\hline $\begin{array}{c}\text { Bivariate Correlations (Pearson } \\
\text { Correlation) }\end{array}$ & 1 & 2 & 3 & 4 & 5 & 6 & 7 & 8 & 9 & 10 \\
\hline 1. Age & 1 & & & & & & & & & \\
\hline 2. Gender & $-.094 * *$ & 1 & & & & & & & & \\
\hline 3. Race & 0.053 & 0.027 & 1 & & & & & & & \\
\hline 4. Education & 0.023 & $-.231 * *$ & $-.094 * *$ & 1 & & & & & & \\
\hline 5. Security/Non-Security & $\begin{array}{c}-.223 * * \\
-\end{array}$ & $.298 * *$ & -0.015 & $-.363^{* *}$ & 1 & & & & & \\
\hline 6. TRAUMA Factor & $\begin{array}{c}0.117 * * \\
-\end{array}$ & -0.001 & 0.018 & -0.019 & $.128 * *$ & 1 & & & & \\
\hline 7. Anxious Arousal & $0.138 * *$ & -0.034 & 0.042 & 0.013 & 0.070 & $.882 * *$ & 1 & & & \\
\hline 8. Intrusive Experiences & $-0.092 *$ & 0.010 & 0.021 & -0.034 & $.124 * *$ & $.935^{* *}$ & $.748 * *$ & 1 & & \\
\hline 9. Defensive Avoidance & $-0.084 *$ & -0.011 & -0.009 & -0.027 & $.115^{* *}$ & $.928 * *$ & $.749 * *$ & $.851 * *$ & 1 & \\
\hline 10. Dissociation & $-.115 * *$ & 0.052 & -0.018 & -0.019 & $.153 * *$ & $.830 * *$ & $.654 * *$ & $.713 * *$ & $.687 * *$ & 1 \\
\hline 11. Depressive Symptoms & -0.028 & -0.055 & 0.032 & 0.005 & $.077 *$ & $.743 * *$ & $.703 * *$ & $.664 * *$ & $.655^{* *}$ & $.649 * *$ \\
\hline 12. Sadness & 0.058 & $-.134 * *$ & -0.027 & $.077 *$ & -0.028 & $.540 * *$ & $.531 * *$ & $.485 * *$ & $.475 * *$ & $.437 * *$ \\
\hline 13. Feeling Worthless & $-0.082 *$ & -0.009 & 0.058 & -0.017 & $.092 *$ & $.631 * *$ & $.593 * *$ & $.552 * *$ & $.546^{* *}$ & $.590 * *$ \\
\hline 14. Avoiding People & -0.039 & -0.005 & 0.044 & -0.039 & $.119 * *$ & $.694 * *$ & $.640 * *$ & $.629 * *$ & $.622 * *$ & $.596 * *$ \\
\hline 15. Suicidal Ideation & -0.048 & $.116^{* *}$ & -0.009 & -0.014 & $.096 * *$ & $.517 * *$ & $.418 * *$ & $.492 * *$ & $.436 * *$ & $.528 * *$ \\
\hline 16. Wishing you were dead & -0.036 & $.076^{*}$ & -0.007 & 0.023 & 0.054 & $.424 * *$ & $.338 * *$ & $.389 * *$ & $.350 * *$ & $.469 * *$ \\
\hline $\begin{array}{l}\text { 17. Fantasies about dying } \\
\text { 18. Feeling so hopeless you wanted to }\end{array}$ & -0.035 & $.107 * *$ & 0.004 & -0.018 & $.079 *$ & $.471 * *$ & $.393 * *$ & $.465^{* *}$ & $.398 * *$ & $.443^{* *}$ \\
\hline & -0.025 & $.090 *$ & -0.007 & -0.041 & $.105^{* *}$ & $.499 * *$ & $.403 * *$ & $.468 * *$ & $.426 * *$ & $.516^{* *}$ \\
\hline 19. Thinking about killing yourself & $-.080 *$ & $.134 * *$ & -0.011 & -0.017 & $.092 *$ & $.396 * *$ & $.314 * *$ & $.383 * *$ & $.336 * *$ & $.399 * *$ \\
\hline
\end{tabular}

$(* *=$ significant at $\mathrm{p}<0.001 ;$ two-tailed $)$

$(*=$ significant at $\mathrm{p}<0.05$; two-tailed $)$ 


\section{Bivariate Correlations (Pearson}

Correlation)

11

11. Depressive Symptoms

$.798 * * \quad 1$

12. Sadness

$.856^{* *} \quad .517 * * \quad 1$

13. Feeling Worthless

14. Avoiding People

$\begin{array}{llll}.867 * * & .535 * * & .627 * * & 1\end{array}$

15. Suicidal Ideation

$\begin{array}{lllll}.631^{* *} & .435^{* *} & .613^{* *} & .536^{* *} & 1\end{array}$

16. Wishing you were dead

$.561 * * \quad .412 * * \quad .539 * * \quad .459 * *$

17. Fantasies about dying

$\begin{array}{lllllll}.516 * * & .339 * * & .501 * * & .452 * * & .838 * * & .575 * * & 1\end{array}$

18. Feeling so hopeless you wanted to die

$\begin{array}{llllllll}.623 * * & .427 * * & .609 * * & .529 * * & .902 * * & .751 * * & .648 * * & 1\end{array}$

19. Thinking about killing yourself

$.485 * * \quad .328 * *$

$.470 * *$

$.416 * * \quad .872 * * \quad .669 * *$

$.680 * * \quad .720 * *$

$(* *=$ Significant at $\mathrm{p}<0.01$, two-tailed $)$

$(*=$ Significant at $\mathrm{p}<0.05$, two-tailed $)$ 
The SEM analysis in this study used three steps. The first step was the use of Confirmatory Factor Analysis (CFA), providing a measurement model of the three latent variables and the observed variables that make them (Higgins et al., 2006; Schreiber, et al., 2006). In the current study, the CFA (Model 1) is meant to test whether the scales and items from the TSI-2's TRAUMA Factor, Depression scale, and Suicidal Ideation subscale are reliably measuring their intended latent variables. In order to assess the construct validity of the latent variables, the model's goodness of fit and the strength of each measurement scale and items' correlation to their intended latent variable (i.e. their factor loadings) were examined (Higgins et al., 2006). In the second step, two models were created to provide evidence that alternative models were necessary to better explain the observed variance in the sample. Model 2 examined whether PTSD and depressive symptoms have independent, direct effects on suicidal ideation and Model 3 examined whether PTSD has an indirect effect on suicidal ideation through its effect on depressive symptoms.

When the findings from step one indicated that the variables were measured accurately and step two provided evidence that some relationship or relationships exist between PTSD, depressive symptoms, and suicidal ideation, the analysis continued to step three. Step three was the creation of an alternative structural model based on input from a prior-existing theory (i.e. General Strain Theory), with the goal of better explaining the relationships seen within the data compared to the models in step two (Higgins et al., 2006). For this study, the alternative model proposed (Model 4) combined the direct and indirect effect models and examined whether depressive symptoms fully 
mediated PTSD's effect on suicidal ideation. According to GST, we would expect for depressive symptoms to be a significant mediator between PTSD and suicidality.

\section{Model 1}

In developing the measurement model, 4 full scales and 2 partial scales were used in forming the latent variables of PTSD, Depressive Symptoms and Suicidal Ideation. The measure for the latent variable of PTSD was formed by using all of the scales from the TSI-2's Trauma factor. Three items from the TSI-2's Depression scale (i.e. the SelfDisturbance Factor) were used as a measure of the latent depressive symptoms variable. Finally, from the Externalization factor, four items from the Suicidality subscale of Suicidal Ideation were used as the observed variables measuring the latent Suicidal Ideation variable. The results of the CFA are presented below in Figure 4.

The results of the CFA found that the measurement model was a good fit to the data. Although the chi-square estimates were significant (chi-square $=186.771, p=0.000$ ), suggesting that the model was a misfit to the data, chi-square's sensitivity to larger sample sizes might lead the results to inaccurately represent the data (Hooper et al., 2008). For this reason, other fit indicators, such as the Comparative Fit Index (CFI), Root Mean Square Error of Approximation (RMSEA), and Weighted Root Mean Square Residual (WRMR), are used to determine the model's goodness of fit. The measurement model's CFI was 0.986 , which is higher than the generally accepted cut-off score of $>0.95$ (Hooper et al., 2008). The model's RMSEA was 0.06, which is higher than a "good" fit indication of $<0.05$, remained within the accepted range of $<0.07$ (Hooper et al., 2008). Finally, the WRMR is recommended to be <0.9-1.0 (Schreiber, et al., 2006); 
the WRMR for this model was well below this at .806. Taken altogether, the goodness of fit indicators suggest that the model was a good fit for the data.

(Figure 4: Measurement Model and CFA Results $(* * *=p<.001))$

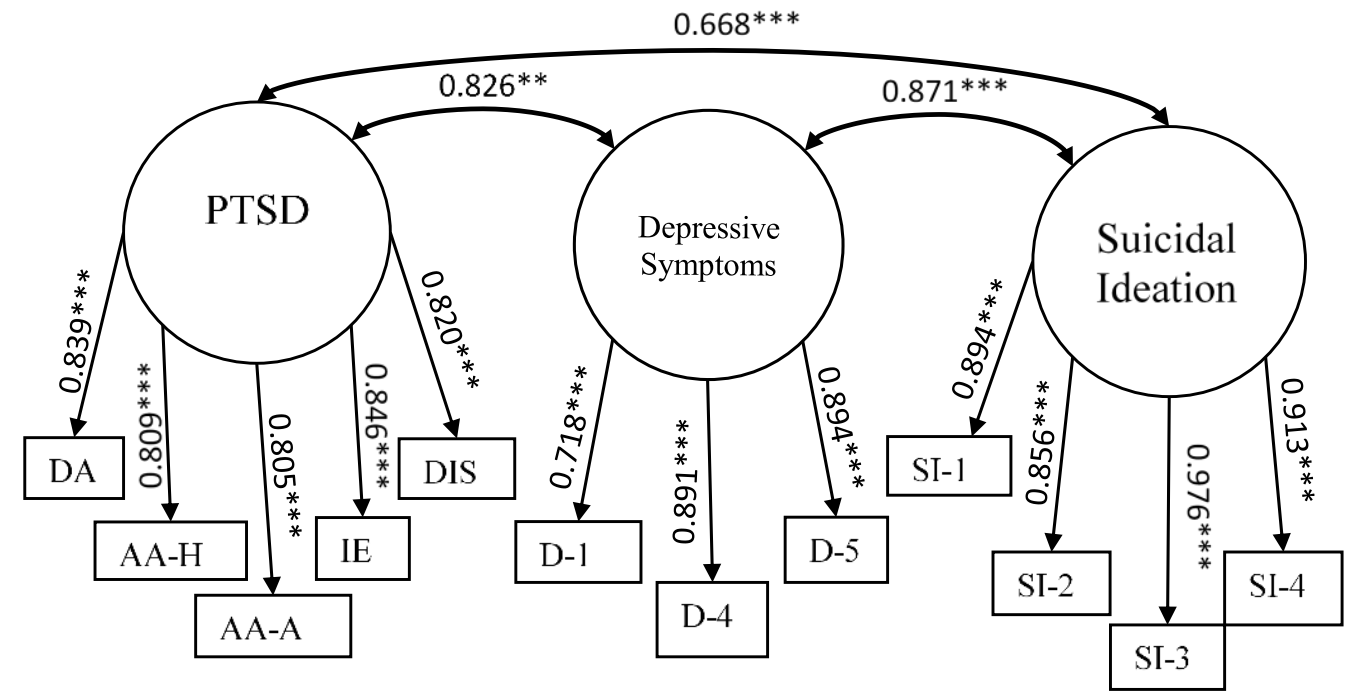

As seen above in Figure 1, the CFA also found that all of the factor loadings for the observed measures were significantly higher than 0.5 , suggesting that the scales and items used to construct the latent variables were valid. In other words, they all were properly measuring their intended latent variables. All measures for the PTSD latent variable had factor loadings above 0.80, with the highest being Intrusive Experiences (0.846) and the lowest being Anxious Arousal-Anxiety (0.805). The three items from the Depression scale that were included in the CFA for Depressive Symptoms reported very high factor loadings (>.70). The TSI-2 item "Sadness" had the weakest factor loading (0.718) of all those in Depressive Symptoms, while the Depression scale item "Feeling worthless" reported the strongest factor loading (0.894). The TSI-2 items used to create the Suicidal Ideation latent construct displayed the strongest factor loadings of all the 
observed variables. The Suicidal Ideation item with the weakest factor loading belonged to the subscale item "fantasies about dying" (0.856). Meanwhile, the item with the strongest factor loading among these observed variables was "feeling so hopeless that you wanted to die" (0.976). Overall, the strength and significance of the factor loadings across all of the observed variables in the measurement model indicated that the items used are acceptable measures of their intended variables.

After having confirmed that each observed variable was measuring its intended construct, the next important piece of information provided by the measurement model was the significant relationships found between the latent variables themselves. All three latent variables were significantly related to each other when no directionality was considered. The strongest relationship was between Depressive Symptoms and Suicidal Ideation (.871) and the weakest relationship was between the latent variables was between PTSD and Suicidal Ideation (.668). A significant relationship was also observed between PTSD and Depressive Symptoms (0.826). The results of the CFA's measurement model suggested that significant but unexplained relationships existed between the latent variables and called for further models in order to examine the directionality and strength of these relationships.

\section{Model 2}

After the CFA confirmed that the items used were correctly measuring their intended latent constructs and that the measurement model was over-identified (i.e. contained multiple unexplained relationships), allowing for structural adjustments to better explain variance, the second structural model was created. In order to determine whether there were any independent, direct relationships between the independent 
variables and suicidal ideation, Model 2 regressed suicidal ideation on both PTSD and Depressive symptoms.

The fit of this direct effects model was the same as the measurement models (chisquare: 186.771, p=.000; CFI: 0.986; RMSEA: 0.06; WRMR: 0.806). In both of these models, the RMSEA value (0.06) was just above the commonly agreed "good" fit threshold (0.05); however, this is still well below what some have discussed as the "mediocre" fit threshold of 0.08 (Hooper et al., 2008; MacCallum, Browne, \& Sugawara, 1996) and all other measures of fit indicate good model fit. Model 2 and its results can be seen below (Figure 4).

Model 2 indicated that PTSD had a very weak and negative, but significant effect, on suicidal ideation (Unstandardized direct effect: $-0.048, \mathrm{p}<0.05, \mathrm{p}=0.023$; Standardized: $-0.161, \mathrm{p}<0.05, \mathrm{p}=0.021)$, independent of depressive symptoms. Depressive symptoms also had a significant positive, direct effect on suicidal ideation, independent of PTSD (Unstandardized direct effect: 1.250, $\mathrm{p}>0.001, \mathrm{p}=0.000$; Standardized: $1.004, \mathrm{p}>0.001, \mathrm{p}=0.000)$. 
(Figure 5: Direct Effects Model $(* * *=p<0.000 ; *=p<0.05))$

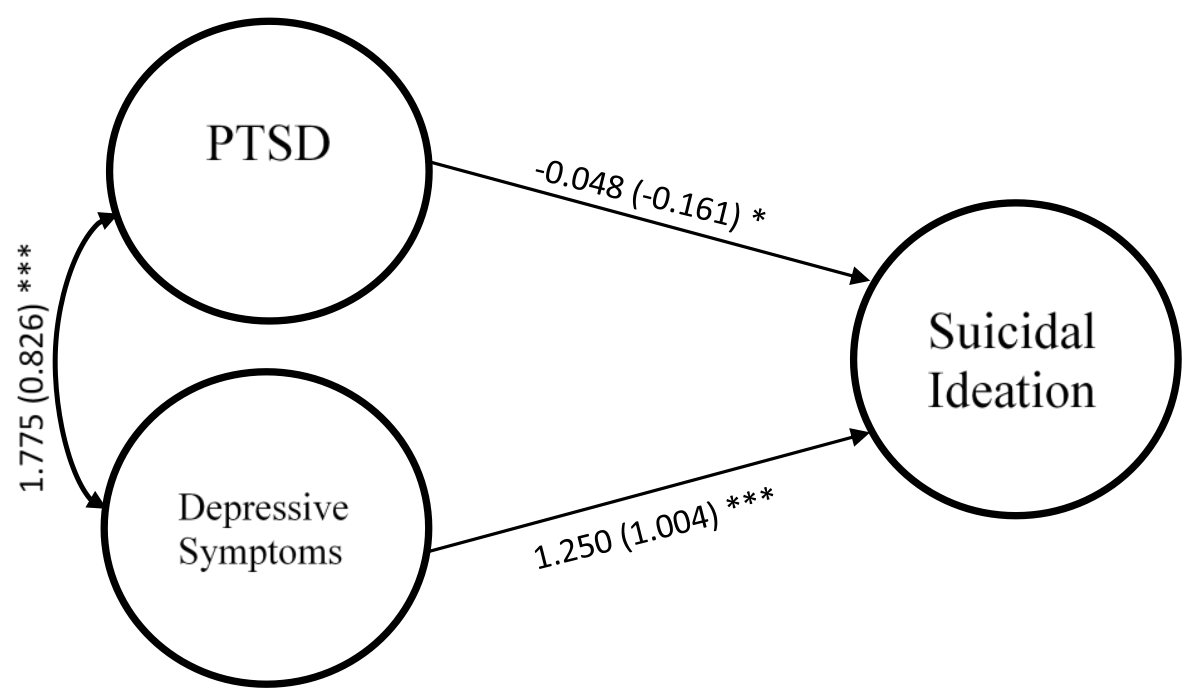

This model indicated that both PTSD and Depressive symptoms are significantly correlated, suggesting a relationship between the two variables that remains unexplained. Model 2 also found significant direct relationships between both PTSD and Suicidal Ideation, and Depressive symptoms and Suicidal Ideation. Given these findings, Model 2 justifies the continued analysis using alternative models.

\section{Model 3}

After finding evidence of the direct effects illustrated in Model 2, the second structural model (Model 3) examined only whether PTSD had an indirect effect on suicidal ideation through depressive symptoms. In order to examine this potential indirect effect, suicidal ideation was regressed onto depressive symptoms, while depressive symptoms were regressed onto PTSD. The first step in determining whether depressive symptoms mediated PTSD and suicidal ideation is identifying that the model is a good fit to the data. The measures of fit for the indirect structural model were not identical to the previous two but were very similar, with the model remaining a good fit to the data (chi- 
square: 191.350, p<0.001, p=.0000; RMSEA: 0.60; CFI: 0.985; WRMR: 0.840). Pictured below are the results from Model 3, the indirect effects model (Figure 6).

(Figure 6: Indirect Effects Model $(* * *=p<0.000)$ )

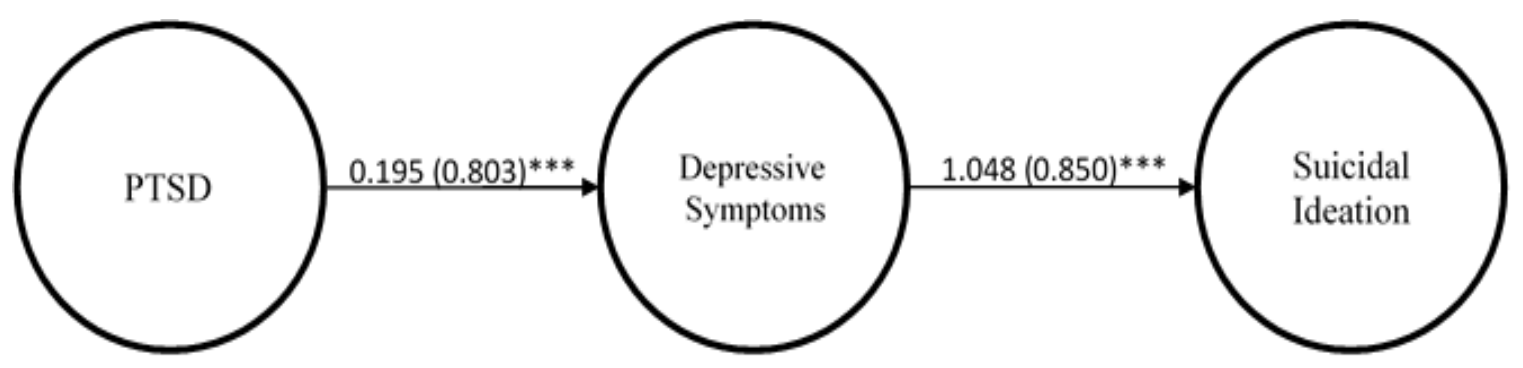

The indirect effects model (Model 3) reported that PTSD had a significant, positive, direct influence on the development of depressive symptoms (Unstandardized direct effect: 0.195, $\mathrm{p}<0.001, \mathrm{p}=0.000$; Standardized: 0.803, $\mathrm{p}<0.001, \mathrm{p}=0.000$ ). Further, depressive symptoms had a strong, positive, direct effect on suicidal ideation (Unstandardized direct effect: 1.048, $\mathrm{p}<0.001, \mathrm{p}=0.000$; Standardized: 0.805, $\mathrm{p}<0.001$, $\mathrm{p}=0.000)$.

Taken together, this shows that PTSD influenced the development of depressive symptoms, which in turn, had a major influence on the development of suicidal ideation. In other words, the results found that PTSD had a positive, indirect effect (Unstandardized: 0.204; Standardized: 0.646) on suicidal ideation, through the development of depressive symptoms. The results of this model provide support for a possible full mediation of PTSD's direct effect on suicidal ideation. Further, these findings are in line with the central argument of GST. GST argues that strain's main effect on anti-social, criminal, or delinquent coping comes through the mediation of a negative affective state, such as anger or depression. 
The results found in Model 3 show support for this and the idea that depressive symptoms can mediate PTSD's relationship with suicidal ideation. PTSD demonstrated a positive, indirect effect on suicidal ideation, which was mediated through PTSD's direct effect on depressive symptoms. In other words, experiencing PTSD (i.e. strain) led to an individual being more likely to develop depressive symptoms (i.e. negative affective state), which in turn made them more likely to experience suicidal ideation (i.e. antisocial coping). The final question, then, is whether PTSD's indirect effect through depressive symptoms fully mediated PTSD's significant, direct effect on suicidal ideation.

\section{Model 4}

The fourth and final model examined whether PTSD's direct effect on suicidal ideation maintained its significance in the presence of PTSD's indirect effect on suicidal ideation through the mediating variable of depressive symptoms. The measures of fit for this model were identical to those found in both the measurement model (Model 1) and the direct effects model (Model 2) (chi-square: 186.771, p>0.001, p=.000; RMSEA: 0.06; CFI: 0.986; WRMR: 0.806). This indicates both that the model is a good fit to the data, and that Model 4 shows a very slight improvement of fit over the indirect effect only model. The results of the direct and indirect effects model are displayed below (Figure 7).

(Figure 7: Direct and Indirect Effects Model $(* * *=p<0.000 ; *=p<0.05)$ ) 


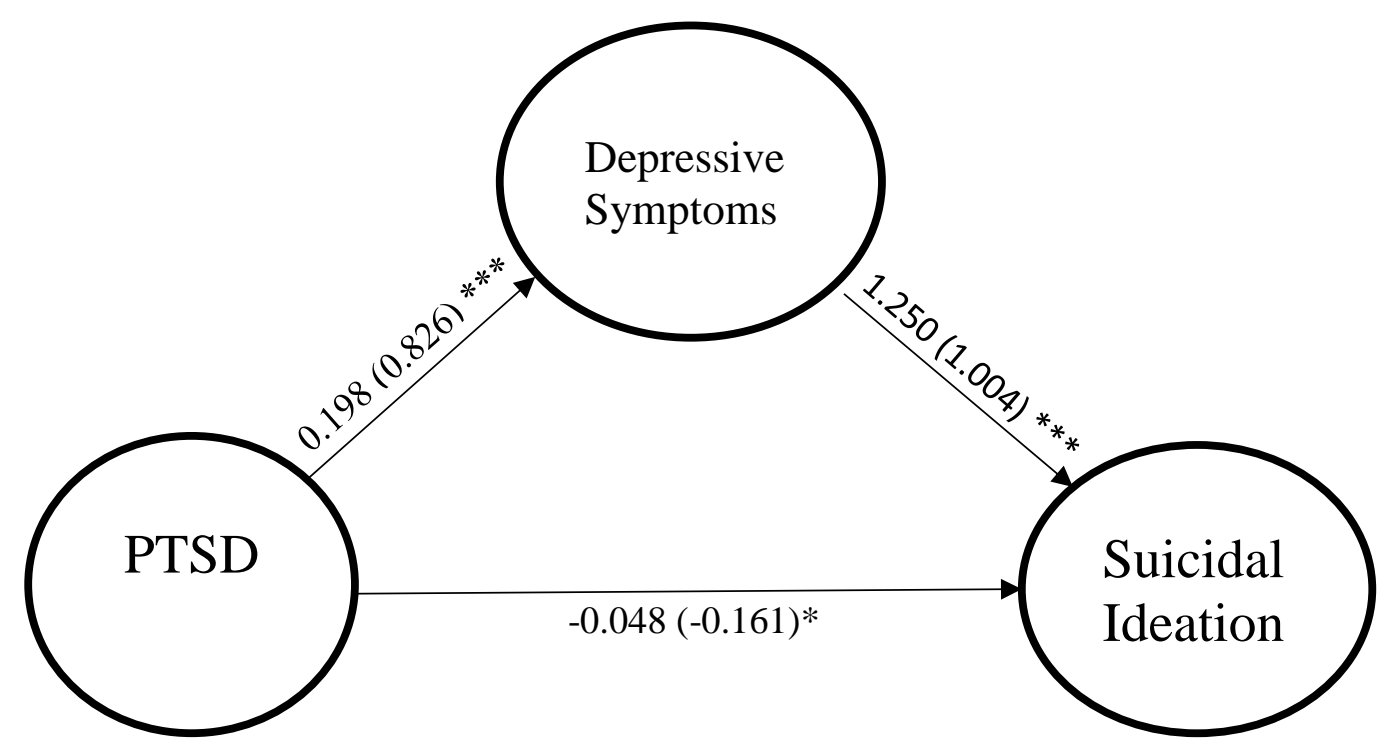

Model 4 indicated that PTSD had a negative but significant direct effect on suicidal ideation (Unstandardized: $-0.048, \mathrm{p}<.05 \mathrm{p}=0.023$; Standardized: $-0.161, \mathrm{p}<.05$, $\mathrm{p}=0.021$ ), while depressive symptoms were seen as having a positive, strongly significant direct effect on suicidal ideation (Unstandardized: 1.250, $\mathrm{p}<.001 \mathrm{p}=0.000$; Standardized: 1.004, $\mathrm{p}<.001, \mathrm{p}=0.000)$. PTSD also had a positive and moderately significant direct effect on the development of depressive symptoms (Unstandardized: 0.198, $\mathrm{p}<.001$ $\mathrm{p}=0.000$; Standardized: 0.826, $\mathrm{p}<.001, \mathrm{p}=0.000)$. The indirect effect of PTSD on suicidal ideation, through depressive symptoms, was found to be moderate to very strong (Unstandardized: 0.248; Standardized: 0.829). This is the second instance in which the model fit was improved from the indirect model, seemingly by the inclusion of the direct effect of PTSD on suicidal ideation.

Observing the total effect or both the indirect and direct effects together, should indicate that depressive symptoms act as a mediating variable between PTSD and suicidal ideation. The negative direct effect between PTSD and suicidal ideation, however, is still seen as significant, despite being mediated by the strong, positive, indirect effect of 
PTSD through depressive symptoms. This leads to a positive total effect of PTSD on suicidal ideation that ranges from 0.20 (Unstandardized) to 0.67 (Standardized). Although the total effect indicated that the direct effect of PTSD on suicidal ideation could be mediated through depressive symptoms, the lingering significance of PTSD's direct effect (weak, negative, or otherwise) remained after the introduction of the mediator. This would indicate that depressive symptoms, no matter how powerful their effect, are only partially mediating the relationship. Possible explanations for this lingering significance and PTSD's negative link with suicidal ideation are discussed later 


\section{CHAPTER 6: DISCUSSION}

This study used a GST framework to investigate the effects of PTSD and depressive symptoms on suicide ideation in a sample of Kentucky Department of Correction's institutional staff, with the purpose of expanding the literature regarding the relationships between PTSD, depression, and suicidality. More accurately, this study examined whether the effects of a strain (i.e. PTSD) on antisocial coping (i.e. suicidal ideation) were mediated by the presence of a negative affective state (i.e. depressive symptoms). Overall, the results provided support for this hypothesis, finding that depressive symptoms significantly mediated the effect PTSD had on increased suicidal ideation. In doing so, the results also provided support for the use of GST as a framework for studying suicide risk in individuals with PTSD, especially those in high-risk populations such as corrections. The findings from this study were supported by the preexisting research; this is discussed below.

A series of measurement and structural models were developed, guided by a GST framework, to observe the significance of any direct, indirect, and/or simultaneous relationships between PTSD, depressive symptoms, and suicidal ideation. The first model examined the independent direct effects of PTSD and depressive symptoms on suicidal 
ideation and revealed that higher PTSD levels led to slightly but significantly lower rates of suicidal ideation $(-0.048[-0.161] ; \mathrm{p}=0.023[0.021] ; \mathrm{p}<.05)$, while higher depressive symptoms were seen as influencing significantly higher reported suicidal ideation (1.250 [1.004]; $\mathrm{p}<.000)$. Depression's observed influence on suicidal ideation is in line with previous findings in regards to both general samples (Cougle et al., 2009a; Krysinska \& Lester, 2010; Smith et al., 2016) and samples of high-risk occupations (Hellmuth et al., 2012; Martin et al., 2017; Stanley et al., 2017). On the other hand, PTSD's relationship with suicidal ideation within the sample was found to be inconsistent with the majority of the literature. While studies including both general samples (Krysinska \& Lester, 2010; LeBouthillier et al., 2015; Panagioti et al., 2015; Poindexter et al., 2015; Rojas et al., 2015; Smith et al., 2016) and samples of high-risk occupations (Boffa et al., 2016; Hellmuth et al., 2012; Martin et al., 2017; Stanley et al., 2017; Violanti, 2004) have consistently reported that PTSD was associated with a higher risk of suicidal ideation, the current study found that PTSD lowered the risk of suicidal ideation.

The second structural model (Model 3) was the GST-inspired model, examining whether strain (i.e. PTSD) indirectly increased the use of maladaptive coping (i.e. suicidal ideation) through its creation of a negative affective state (i.e. depressive symptoms) (Agnew 1992, 2001, 2006; Agnew, et al., 2002). GST states that strains, or life stressors, lead to maladaptive coping when individuals seek a means of relieving the negative affective state caused by the strain. However, not everyone responds to stressful life events or relationships with maladaptive coping. This led Agnew (1992) to propose that individuals experienced unique emotional responses to different stressors, which he called "subjective strain." When these emotional responses are intense and negative, they 
tend to create pressure for coping behaviors, which, depending on an individual's access to social support, pro-social coping resources/skills, and previous coping history, could include antisocial coping behaviors such as suicidal ideation or behavior.

Overall, the main model testing GST's statements (Model 3) found support for the use of the theory as a viable framework, with findings revealing that the relationship between strain (i.e. PTSD) and maladaptive coping (i.e. Suicidal Ideation) that was strongly mediated through a negative affective state brought on by the strain. PTSD indirectly increased suicidal ideation through causing higher rates of depressive symptoms, which in turn directly increased suicidal ideation. Not only is this in line with GST's statements that strain on its own is often insufficient in causing antisocial coping and that this relationship is instead mediated by the development of a negative effective state (Bishopp \& Boots, 2014; Lin, Cochran, \& Mieczkowski, 2011). These findings are also supported by the existing research on the mediating effects of depressive symptoms on PTSD's relationships with suicidal ideation in both general (Krysinska \& Lester, 2010; Panagioti et al., 2015; Poindexter et al., 2015) and high-risk samples (Hellmuth et al., 2012; Stanley et al., 2017).

As indicated in the final structural model (Model 4), both the direct and indirect effects between PTSD and suicidal ideation were tested together. Although PTSD's effect on suicidal ideation was much stronger indirectly through its relationship with depressive symptoms, PTSD continued to negatively influence suicidal ideation on its own, which indicated strong, but not total, mediation. Some studies have found that subthreshold depression- or experiencing fewer depressive symptoms than required for diagnosis- can fail to fully mediate PTSD's relationship with suicidal ideation and behavior (Panagioti et 
al., 2015; Poindexter et al., 2015). This may explain this lingering significance, as only three depressive symptoms were included in the analysis. Still, the mediation that was seen from these symptoms suggest that PTSD's relationship with suicidal ideation could be best explained as PTSD increasing experienced depressive symptoms, which increases suicidal ideation. Not only was this finding consistent with the existing research (Krysinska \& Lester, 2010; Panagioti et al., 2015; Poindexter et al., 2015), but it also supported GST's viability as an applicable framework for examining suicide risk among individuals with clinically elevated PTSD symptoms. To summarize, in this sample, strain, measured as PTSD symptomology directly decreased rates of maladaptive coping (i.e. suicidal ideation), but indirectly increasing suicidal ideation when PTSD led to the development of a negative affective state (i.e. depressive symptoms), which ultimately led to an increase in maladaptive coping behaviors. Again, the results from this model provided support for GST's use in examining the relationship between PTSD, depressive symptoms, and suicidal ideation.

The majority of the findings in this study are consistent with the greater body of research on the relationships between PTSD, depression, and suicidality. One finding within this study did stand out as inconsistent: The weak, negative direct effect PTSD displayed suicidal ideation. Although some studies have found that PTSD is a weak predictor of suicide on its own (Cougle et al., 2009a; Krysinska \& Lester, 2010), or that not all depressive symptoms are capable of fully mediating the effects of PTSD on suicidal ideation (Panagioti et al., 2015; Poindexter et al., 2015), none of the literature previously reviewed reported PTSD as significantly decreasing suicide risk. There are a few plausible reasons for the finding within this sample. One potential explanation is that 
the findings accurately represent the relationship within the data: Something about the PTSD scores in this sample initially causes a very slight reduction in levels of suicidal ideation before considering any mediating variables. One possibility for this may be that the data accurately showed PTSD as decreasing suicidal ideation through the observed variables forming PTSD's latent construct. The inverse causal relationship observed between PTSD and suicidal ideation was the weakest of any of those identified through the analyses. It is plausible, then, that rather than being the entire construct that was exhibiting this weak but significant direct effect, it instead may reflect the underlying, independent or collective effect of a specific representation of the TRAUMA factor scales.

To explain further, one study in the preexisting research highlighted that there are over a half-million ways that PTSD symptoms can be expressed before considering symptoms from comorbid disorders (Young et al., 2014). These different representations (i.e. varying combinations of symptoms and symptom levels) vary across populations and the types of trauma exposure, influencing different observed outcomes across different samples. Other research has indicated that varying levels of PTSD's re-experiencing and numbing symptoms clusters (i.e. the symptoms measured by the TSI-2's IE and DIS scales) display the only significant effects among PTSD symptoms on heightened suicidal ideation and attempts in high-risk samples (Boffa et al., 2017; Hellmuth et al., 2010; Stanley et al., 2017). At the same time, the TSI-2's TRAUMA factor includes the Anxious Arousal (AA) scale, which contains items that seem to be in total contrast to the items from the Suicidality-Ideation (SUI-I) subscale. For example, the AA scale includes "Feeling afraid of certain things, even when there probably wasn't any danger," "feeling 
afraid you might die or be injured" and "watching out for danger," while the SUI-I scale's items measure experiences such as "Fantasies about dying" and "wishing you were dead" (Briere, 2011). As these items seem to be measuring opposite experiences, it is plausible that they could display a significant negative relationship with one another.

The weak negative influence of PTSD on suicidal ideation, then, could potentially be attributable to a novel PTSD symptom combination of low IE or DIS scores and/or abnormally high scores in both AA subscales within the sample. As this relationship occurs only when not considering symptoms from other disorders (i.e. Depression), this could also be one reason some studies find that PTSD alone does not increase suicidal ideation or behavior (Cougle et al., 2009a; Krysinska \& Lester, 2010). Few empiricallysupported explanations exist for the inconsistent findings; however, they could also be explained as being design limitations that damaged the outcome of the analysis.

\section{Limitations of the Current Study}

Another potential explanation for the strange results regarding PTSD's direct, negative effect could come from one major issue in the study design, which may have influenced the analysis. The biggest limitation experienced in this process was the exclusion of TSI-2 scale items in forming the latent depression variable and the observed suicidal ideation variable due to their high levels of multicollinearity. The specific software used to conduct the SEM analysis (MPLUS 3.1) is especially sensitive to highly correlated observed measures when constructing the latent variables. This feature is meant to assure the construct validity of the proposed latent variables by being highly discriminate in the level collinearity it allows. This led to issues, however, when using a 
single measurement tool designed to measure the symptoms commonly experienced together (i.e. the TSI-2).

Exclusively using scales from Briere's (2011) TSI-2 (and not anticipating their high levels of multicollinearity) led to MPLUS's exclusion of a significant number of observed measures intended for use in forming the latent Depression and the observed suicidal ideation variables. In total, seven of the ten depression scale items, one the five suicidal ideation items, and all measures of suicidal behavior were excluded from the study. While some of these exclusions might not have been avoidable, it should be noted that these items were discarded, rather than excluding any of the items in the TRAUMA scales, in order to maintain an accurate measure of PTSD symptoms.

As mentioned above, one limitation to the study was that some items were excluded to assure the TRAUMA factor scales were retained in their entirety. Keeping some of these measures came at the cost of excluding a majority of the depression scale items. Specifically, the lack of TSI-2 Depression scale items used in forming the latent construct for depressive symptoms was due in large part to retaining the full AA-Anxiety scale and any of the suicidal ideation scale. Although the CFA revealed that each observed measure (i.e. TSI-2 item) was measuring only it's intended latent variable, it is possible that by not including all of the distinct depression symptoms measured in the TSI-2, some of the depression symptoms that are shared with PTSD may have been measured inadvertently with that latent construct.

To elaborate, the latent variable of depressive symptoms was constructed using only three TSI-2 depression scale items: reported levels of "sadness," "being so depressed you avoided people," and "feeling worthless" (Briere, 2011). These exclusions 
were originally thought to limit only the ability to describe the latent mediating variable as “depressive symptoms," as opposed to "depression”. In retrospect, however, not controlling for depression symptoms that are also commonly seen as symptoms in PTSD diagnoses (i.e. decreased attention/zoning out, loss of appetite, insomnia, anhedonia, etc.) could be another possible reason for PTSD's lingering significant direct effect. If the lack of depression measures used in the forming of the latent variable meant that some depression symptoms that are shared with PTSD went unmeasured, the significance of PTSD's direct effect on suicidal ideation might have existed because these excluded depression symptoms were measured instead in the responses to similar PTSD items.

Three of the five TSI-2 TRAUMA symptom scales include an item that could measure both a PTSD and depression symptom that is not controlled for: The AAHyperarousal subscale includes a direct measure of the shared depression symptom of insomnia. Anhedonia (i.e. no longer enjoying activities/places/people you used to enjoy) is included in the Defensive Avoidance scale. Finally, the Dissociation scale includes measures of increased inattention. While these commonly shared symptoms may not be directly related to suicidal ideation, it is plausible that the failure to include a control for them in the latent depression construct could have resulted in depression's partial, rather than total, mediation of the significance of PTSD's effect on suicidal ideation.

Another possible issue caused by the limitations of excluding a large portion of observed measures in forming the observed suicidal ideation variable. In measuring suicidal ideation only, rather than suicidality as a whole, this study may have missed the exact nature of the relationship that exists between PTSD, depression, and suicidality. The results indicated that PTSD decreased suicidal ideation when not considering the 
depressive symptoms caused by PTSD. However, some studies have suggested that depression is only responsible for the experiencing of suicidal ideation and that it takes some type of catalyst, such as PTSD, to incite suicidal behavior (Cougle et al., 2009; Krysinska \& Lester, 2010). The results of this study could show that PTSD has no hand in increasing suicidal thoughts, but further research is needed to determine if depression mediates PTSD's effects on suicidal behavior. The next section discusses ways these issues might be avoided in the future, as well as other implications from the findings for future research and policy.

\section{Implications for Future Research/Policy}

There are many implications that can be drawn from the results that may be of interest for future researchers interested in replicating this study or expanding the literature on PTSD, depression, and suicidality. The results also have implications for uses of General Strain Theory and for new policies for correctional staff and other highrisk occupations. In order to avoid the major limitation of high multicollinearity discussed in the previous section, future researchers should use multiple measurement tools in forming the latent and observed variables used in the models. If the TSI-2 is used as the only measurement tool again, researchers should allow for the exclusion of items from the TRAUMA Factor that are either measured as part of other TSI-2 Factor scales or measure symptoms shared with other disorders (i.e. not unique to PTSD).

Beyond changes to the design of future replications of this study, one persistent theme among the findings in this study (and throughout the literature on PTSD and suicidality) was the need for further investigation into the unique relationships between PTSD's individual symptom clusters and suicidality. Although studies on this subject 
exist focusing on veterans (Hellmuth et al., 2010) and firefighters (Boffa et al., 2016; Stanley et al., 2017), none were found examining correctional staff, correctional officers, or any other law enforcement positions, presenting a large gap in the existing research.

The findings from the studies into PTSD symptom clusters and suicidality which do exist all suggest that these clusters exhibit their own individual effects on suicidalityrelationships which can even be mediated by depression (Boffa et al., 2016; Hellmuth et al., 2010; Stanley et al., 2017). Future studies could examine if these relationships and representations of PTSD are found across all types of high-risk samples. To further investigate the anomalous finding within this study, future research could examine whether the significant negative relationship is observed among any of the individual symptom clusters or TSI-2 TRAUMA factor scales. Other potential future research grounded in these findings could examine whether the same results found for depression mediating suicidal ideation are seen among suicidal behavior.

The results of this study carry a number of implications for Agnew's (1992) General Strain Theory and its use in examining the relationships between PTSD, depression, and suicidality. Mainly, the results confirm that GST is a viable framework for exploring and understanding the relationships between these constructs. Strain is most likely to lead to antisocial or delinquent coping when that strain causes a negative affective state. This negative affective state is what creates the drive for some form of coping; depending on the individual's resources and access to proper coping mechanisms, they may resort to antisocial coping (e.g. suicidal ideation or behavior) to alleviate it (Agnew 1992, 2001, 2006; Agnew, et al., 2002). The results of this study found almost exactly that: PTSD, used as an analog for trauma, (i.e. the strain) was found to not be a 
cause of suicidal ideation (i.e. antisocial coping) on its own. Instead, PTSD's strongest influence over suicidal ideation was seen indirectly, through the depressive symptoms (i.e. negative affective state) caused by PTSD symptoms. In other words, PTSD's influence over suicidal ideation was mediated by the creation of depressive symptoms. Along with confirming the use of a GST framework, the results of this study also add to the GST literature by displaying that depression is a viable mediator for antisocial behavior. While most studies tend to focus on anger as a mediator (Mazerolle et al., 2003; Rebellan et al., 2012; Rebellan et al., 2009), the few that include measures of different negative affective states, such as anxiety (Aseltine et al., 2000), tend to find results in line with those of the current study. That is, depression can act as the mediating emotion that causes antisocial, if not delinquent or criminal, coping behaviors (Bishopp \&Boots, 2014; Krysinska \& Lester, 2010; McKinney et al., 2017; Panagioti et al., 2015; Poindexter et al., 2015). It is also this finding, that depression mediates PTSD's effects on suicidal ideation, that should be of most concern to those in charge of policy for the safety of those working in high-risk occupations, particularly those working as correctional staff.

The finding that depressive symptoms were the cause of suicidal ideation among KY DOC staff with high PTSD symptomology has strong implications for the policies of high-risk work environments. It has been seen that both direct and indirect exposure to violence or death in the workplace can lead to the development of PTSD in samples of police officers (Marmar et al., 2006; Robinson et al., 1997), EMTs (Marmar et al., 1996), fire fighters (Boffa et al., 2016; Stanley et al., 2017) and correctional staff (Denhof \& Spinaris, 2013, 2016; Spinaris et al., 2012). The results of this study suggest that both direct and indirect workplace traumas (measured as PTSD symptom levels of security 
and non-security correctional staff) increased suicidal ideation indirectly, through the development of depressive symptoms caused by PTSD. If these depressive symptoms were the main source of suicidal ideation, then learning to prevent, recognize, and treat these depressive symptoms should be a priority in forming future policies to help prevent suicides and treat the more dangerous aspects of workplace-related PTSD. One possible suggestion would be for the DOC to implement mandatory trauma-related CognitiveBehavioral Therapy following Violence, Injury, or Death events. Cognitive Behavioral Therapy (CBT) is a subset of therapeutic interventions centered around confronting (and thereby altering) the maladaptive cognitions and their related behaviors caused by disorders. By targeting the specific behaviors and thoughts caused by disorder symptoms, CBT is not only highly customizable to the individuals or groups in therapy but is also highly effective in treating a wide range of behaviors and disorders that could benefit employees of the DOC (Hofmann et al., 2012). While CBT can be used to target anger and aggression, chronic stress, and even PTSD itself, the most important focus for use by the DOC should be CBT's that can be administered a group setting which target depression. By making sessions mandatory for workers, even those who have not directly experienced a VID event, it could go a long way in reducing the depression caused by work-related PTSD and the increased suicidal ideation that comes with it.

While these results might be applicable to all high-risk occupations, they are more crucial and generalizable to correctional facilities. This is not only because the sample was representative of KY DOC correctional staff, but also because correctional staff have been found to consistently report a higher prevalence of PTSD and depression (Denhof \& Spinaris, 2013, 2016; Spinaris et al., 2012) than most other high-risk occupations, not 
including military veterans (Boffa et al., 2016; Marmar et al., 1996; Marmar et al., 2006; Robinson et al., 1997; Stanley et al., 2017). Another earlier study indicated that correctional officers (specifically) have also significantly elevated suicide rates compared to the general population (Stack \& Tsoudis, 1997), speculating that one potential cause for these rates were the work environment to which correctional officers were exposed. The authors recognized that very little research existed relating to this problem, and that instead most research and outreach regarding suicide in the corrections system is focused on the problem of inmate suicides. This study aimed to fill a small part of that large gap in the research and provide insights for future policy in dealing with preventing correctional staff suicides.

By testing a theoretically-driven explanation of the relationships of PTSD, depression and suicide among a sample of correctional staff, the current study was one of the first to examine the relationships between work-place trauma, mental illness, and high suicide rates among correctional staff (Tiesman et al., 2010). The results should indicate to correctional facilities that, although exposure to violence or death in the workplace alone was not the direct cause for increased suicide risk (i.e. suicide risk), it was found to increase rates of depressive symptoms within the sample. These depressive symptoms, in turn, increased the rates of suicidal ideation. Therefore, VID events should be treated as though they directly increase suicide risk, with policies being put in place, which focus on reducing both the development of PTSD symptoms through limiting exposure to violence and reducing depressive symptoms by helping individual seek treatment when they do experience a traumatic event. As reducing and preventing institutional suicide (i.e. suicides of those in prison/jail) is a major focus among facilities, correctional staff 
often receive training in detecting the warning signs of suicide or depression in the inmates they work around. One major implication from the findings that could help in preventing the suicide of correctional staff would be to include in future training that these signs should be watched for in co-workers. This is just one small change in training that could go a long way in preventing future suicides among correctional staff; however, any future policies should consider the role depressive symptoms play in increasing suicidal thoughts following exposure to a VID event resulting in PTSD. The following section is a summary of the current study and its findings.

\section{Conclusion}

As the fourth highest leading cause of death for individuals ages 18-65 in the United States (National Center for Health Statistics [NCHS], 2016), suicide is a growing and complex problem. There is already a vast body of research that suggests Posttraumatic Stress Disorder and Depression are two major correlates to reporting suicidality. While this was found to be especially true among occupations at a high risk for trauma exposure, such as military veterans, police, and firefighters, the lack of studies examining whether rates of suicidality were higher among correctional staff presented itself as a gap in the research.

Much like other high-risk occupations, correctional staff have been found to report higher rates of PTSD and Depression related to workplace traumas than are found in the general population (Denhof \& Spinaris, 2013, 2016; Spinaris et al., 2016). Despite their reported PTSD and Depression prevalence being greater than that of all other highrisk occupations reviewed (excluding military veterans) (Boffa et al., 2016; Marmar et al., 1996; Marmar et al., 2006; Robinson et al., 1997; Stanley et al., 2017), very few 
studies have examined whether correctional staff see increased rates of suicidality among correctional staff. The purpose of this study was to fill this existing gap in the literature by providing a potential theoretical framework to examine the relationships between PTSD, depression and suicidality. Using a framework guided by General Strain Theory, this study examined one potential explanation for the relationship between PTSD (an analog for workplace trauma), Depressive symptoms (a weakened measure of Depression), and increased suicide ideation (a measure of general suicide risk). This framework specified that the strain (i.e. trauma measured as PTSD) is not sufficient to cause antisocial coping (i.e. suicidal ideation) on its own. Instead, it is when the strain creates a negative affective state (i.e. depressive symptoms) that an individual seeks antisocial coping mechanisms to cope with the strain (Agnew 1992, 2001, 2006; Agnew, et al., 2002). In other words, this study examined whether depressive symptoms were capable of mediating the relationship between PTSD and suicidal ideation.

Although further analysis is needed to confirm the results, this study's findings contained several major implications for both the problem of PTSD in corrections, as well as for the literature on PTSD, Depression and Suicidality. The hypothesis that the GST framework was a viable option for examining PTSD, depression and suicidality was supported. PTSD was seen decreasing suicidal ideation within the sample, which fit the GST framework only in the way that the strain did not cause antisocial coping; it did display a significant, negative relationship, however. PTSD's relationship with suicidal ideation was found to be mediated by the presence of the depressive symptoms it caused; this finding was the strongest support for the use of GST in examining the relationships between PTSD, depression, and suicidality. The results of this study also suggested that 
one possible cause for the inconsistent significant relationship found between PTSD and suicidality in some studies was this mediating relationship (Chen et al., 2017; Cougle et al., 2009a). As depression is the most common disorder associated with suicide and is commonly found to be comorbid with PTSD and other mental disorders, it is possible that this result indicates that depression is a priming agent for suicidality. That is, suicidal ideation may itself be a symptom of depression that, when experienced alongside the symptoms of another disorder, catalyzes further into suicidal behavior.

Finally, these results have implications for the problem of mental health among correctional staff. Mainly, this study bridges the gap between two small bodies of literature: One pertaining to PTSD and Depression among corrections, and the other to high rates of suicide among correctional officers. The results of this study confirm that the high rates of PTSD seen in studies by Denhof, Spinaris and colleagues $(2012,2013$, 2016) are related to the high rates of suicide found in Stack and Tsoudis' (1997) study. However, rather than being the direct cause of suicidal ideation, PTSD was seen as decreasing it, unless it was otherwise mediated by the development of depressive symptoms. This suggests that the high rates of depression seen among correctional staff may not be the direct result of any trauma exposure at all, but are the results the high rates of PTSD, instead. While the results require further testing to determine their accuracy, their one major implication is clear: The workplace violence experienced by correctional staff is directly responsible for increased issues in their mental health, specially increasing rates of PTSD, which are creating higher rates of depressive symptoms and, in the end, suicide. 


\section{REFERENCES:}

Agnew, R. (1985) A Revised Strain Theory of Delinquency. Social Forces, 64(1).

Agnew, R. (1992). Foundation for a general strain theory of crime and delinquency. Criminology, 30(1), 47-87.

Agnew, R. (2001). Building on the Foundation of General Strain Theory: Specifying the Types of Strain most likely to lead to Crime and Delinquency. Journal of Research in Crime and Delinquency, 38(4) 319-361. doi: $10.1177 / 0022427801038004001$

Agnew, R. (2012). Reflection on "A Revised Strain Theory of Delinquency". Social Forces 91(1) 33-38. doi: 10.1093/sf/sos 117

Agnew, R., Brezina, T., Wright, J.P., \& Cullen, F.T. (2002). Strain, Personality Traits, and Delinquency: Expanding General Strain Theory. Criminology 40 (1) 47-71.

Agnew, R. \& White, H. R. (1992) An Empirical Test of General Strain Theory. Criminology 30, pp. 475-499.

American Psychiatric Association. (2013). Diagnostic and Statistical Manual of Mental Disorders (5th ed.). Arlington, VA: American Psychiatric Publishing 
Anderson, R.E., Geier, T.J., \& Cahill, S.P. (2016). Epidemiological associations between posttraumatic stress disorder and incarceration in the National Survey of American Life. Criminal Behavior and Mental Health (26) pg. 110-123. DOI:10.1002/cbm.1951

Aseltine, R.H., Gore, S., \& Gordon, J. (2000) Life Stress, Anger and Anxiety, and Delinquency: An Empirical Test of General Strain Theory. Journal of Health and Social Behavior 41, 256-275.

Asimakopoulou, E. \& Madianos, M. (2014) The Prevalence of Major Depression-PTSD Comorbidity among ICU Survivors in Five General Hospitals of Athens: A Crosssectional Study. Issues in Mental Health Nursing, 35(12), 954-963. doi:

$10.3109 / 01612840.2014 .924609$

Bao, W.N., Haas, A., \& Pi, Y. (2007) Life Strain, Coping, and Delinquency in the Peoples' Republic of China: An Empirical test of General Strain Theory from a Matching Perspective of Social Support. International Journal of Offender Therapy and Comparative Criminology 51(1), pp. 9-24. DOI: $10.1177 / 0306624 X 06294428$

Baron, S. (2004) General Strain, Street Youth and Crime: A test of Agnew's Revised Theory. Criminology, 42(2), pp. 457-484 DOI: 10.1111/j.1745-9125. 2004.tb00526.x

Baron, S.W. \& Hartnagel, T.F. (2002) Street Youth and Labor Market Strain. Journal of Criminal Justice,30 pp. 519-533

Bebbington P., Dunn, G., Jenkins, R., Lewis, G., Brugha, T., Farrell, M., \& Meltzer, H. (2003). The influence of age and sex on the prevalence of depressive conditions: 
report from the National Survey of Psychiatric Morbidity. International Review of Psychiatry, 15(1-2), pp. 74-83. DOI: 10.1080/0954026021000045976

Bishopp, S. A., \& Boots, D. P. (2014). General strain theory, exposure to violence, and suicide ideation among police officers: A gendered approach. Journal of Criminal Justice, 42(6), 538-548. doi:10.1016/j.jcrimjus.2014.09.007

Boffa, J.W., Stanley, I.H., Hom, M.A., Norr, A.M., Joiner, T.E., \& Schmidt, N.B. (2016). PTSD symptoms and suicidal thoughts and behaviors among firefighters. Journal of Psychiatric Research, 84, pp. 277-283.

Breslau, N. (2002). Epidemiologic studies of trauma, posttraumatic stress disorder, and other psychiatric disorders. Canadian Journal Psychiatry/Review Canadian Psychiatry, 47 (10), pp. 923-929

Breslau, N. (2009). The Epidemiology of Trauma, PTSD, and other Post-trauma Disorders. Trauma, Violence, \& Abuse 10(3), pp. 198-210. DOI: $10.1177 / 1524838009334448$

Breslau, N. \& Peterson, E.L. (2010). Assaultive violence and the risk of posttraumatic stress disorder following a subsequent trauma. Behaviour Research and Therapy 48(10), pp. 1063-1066. https://doi.org/10.1016/j.brat.2010.07.001

Breslau, N., Davis, G.C., Peterson, E.L., \& Schultz, L.R. (2000). A second look at comorbidity in victims of trauma: the posttraumatic stress disorder-major depression connection, Biological Psychiatry, 48(9) pp. 902-909 https://doi.org/10.1016/S0006-3223(00)00933-1.

Brezina, T. (1996) Adapting to Strain: An Examination of Delinquent Coping Responses. Criminology, 43(1), pp. 39-61 
Broidy, L.M. (2001) A Test of General Strain Theory. Criminology, 39(1), pp.9-36. DOI: 10.1111/j.1745-9125.2001.tb00915.x

Chen, C.M., Yoon, Y.H., Harford, T.C., \& Grant, B.F. (2017). Dimensionality of DSM-5 posttraumatic stress disorder and its association with suicide attempts: results from the National Epidemiologic Survey on Alcohol and Related Conditions-III. Social Psychiatry and Psychiatric Epidemiology, 52(6), pp. 715-725. DOI: $10.1007 / \mathrm{s} 00127-017-1374-0$

Cloward, R.A. \& Ohlin, L.E. (1960) Delinquency and Opportunity: A theory of Delinquent Gangs. New York: Free Press

Cohen, A.K. (1955) Delinquent Boys. New York: Free Press.

Coronas, R., García-Parés, G., Viladrich, C., Santos, J. M. and Menchón, J. M. (2008) Clinical and sociodemographic variables associated with the onset of posttraumatic stress disorder in road traffic accidents. Depression and Anxiety (25), pp. 16-23 DOI:10.1002/da.20324

Cougle, J. R., Resnick, H., \& Kilpatrick, D. G. (2009)A PTSD, depression, and other comorbidity in relation to suicidality: Cross-sectional and prospective analyses of a national probability sample of women. Depression and Anxiety, 26(12).

Cougle, J.R., Resnick, H. \& Kilpatrick D.G. (2009)B. Does prior exposure to interpersonal violence increase risk of PTSD following subsequent exposure? Behavioral Research and Therapy 47(12), pp. 1012-1017 https://doi.org/10.1016/j.brat.2009.07.014 
Cullen, F., Unnever, J.D., Hartman, J.L., Turner, M.G., \& Agnew, R. (2008) Victims and Offenders Gender, Bullying Victimization, and Juvenile Delinquency: A Test of General Strain Theory. Victims and Offenders, 3(4) pp. 1-29.

Denhof, M.D. \& Spinaris, C.G. (2013) Depression, PTSD, and Comorbidity in United States Corrections Professionals. Desert Waters Correctional Outreach. http://desertwaters.com/wp-content/uploads/2013/09/Comorbidity_Study_09-03131.pdf

Denhof, M.D. \& Spinaris, C.G. (2016). Prevalence of Trauma-related Health Conditions in Correctional Officers: A Profile of Michigan Corrections Organization Members. Desert Waters Correctional Outreach.

Dorrington, S., Zavos, H., Ball, H., McGuffin, P., Rijsdijk, F., Siribaddana, S., Sumathipala, A., \& Hotopf, M. (2014). Trauma, post-traumatic stress disorder and psychiatric disorders in a middle-income setting: prevalence and comorbidity. The British Journal of Psychiatry, 205(5), pp. 383-389 DOI:

10.1192/bjp.bp.113.141796

Follette, V.M., Polusny, M.M., \& Milbeck, K. (1994). Mental Health and Law Enforcement Professionals: Trauma History, Psychological Symptoms, and Impact of Providing Services to Child Sexual Abuse Survivors. Professional Psychology: Research and Practice (25)3, pp. 275-282.

Gradus, J.L. (2017) PTSD and Death from Suicide. PTSD Research Quarterly 28(4). Hellmuth, J.C., Stappenbeck, C.A., Hoerster, K.D., \& Jakupcak, M. (2012) Modeling PTSD Symptom Clusters, Alcohol Misuse, Anger, and Depression as They Relate 
to Aggression and Suicidality in Returning U.S. Veterans. Journal of Traumatic Stress, 25(5). doi: 10.1002/jts.21732

Higgins, G.E., Fell, B.D., \& Wilson, A.L. (2006). Digital Piracy: Assessing the Contributions of an Integrated Self-Control Theory and Social Learning Theory Using Structural Equation Modeling. Criminal Justice Studies, 19(1), pp. 12-15. DOI: $10.1080 / 14786010600615934$

Higgins, G. E., Piquero, N. L., \& Piquero, A. R. (2011). General Strain Theory, Peer Rejection, and Delinquency/Crime. Youth \& Society, 43(4), 1272-1297. doi:10.1177/0044118X10382032

Hofmann, S.G., Asnaani, A., Vonk, I.J.J., Sawyer, A.T., and Fang, A. (2012) The Efficacy of Cognitive Behavioral Therapy: A Review of Meta-analyses. Cognitive Therapy and Research 36(5), pp. 427-440 doi: 10.1007/s10608-012-9476-1]

Ilgen, M.A., Bohnert, A.S.B., Ignacio, R.I., McCarthy, J.F., Valenstein, M.M., Kim, H.M., \& Blow, F.C. (2010). Psychiatric Diagnoses and Risk of Suicide in Veterans. Arch Gen Psychiatry, 67(11). DOI:

10.1001/archgenpsychiatry.2010.129

Kessler, R.C., Berglund, P., Demler, O., Jin, R., Koretz, D., Merikangas, K.R., Rush, A.J., Walters, E.E., Wang, P.S., (2003). The Epidemiology of Major Depressive Disorder Results from the National Comorbidity Survey Replication (NCSR). JAMA, 289(23), pp. 3095-3105. DOI: 10.1001/jama.289.23.3095

Kessler, R.C., Sonnega, A., \& Bromet, E. (1995) Post Traumatic Stress Disorder in the National Comorbidity Survey. JAMA Psychiatry, 52(12). doi:10.1001/archpsyc.1995.03950240066012 
Kilpatrick, D. G., Resnick, H. S., Milanak, M. E., Miller, M. W., Keyes, K. M., \& Friedman, M. J. (2013). National estimates of exposure to traumatic events and PTSD prevalence using DSM-IV and DSM-5 criteria. Journal of Traumatic Stress, 26(5). DOI: 10.1002/jts.21848

Krysinska, K. \& Lester, D. (2010). Post-traumatic stress disorder and suicide risk: a systematic review. Archives of Suicide Research 14(1) pp. 1-23

Langhinrichsen-Rohling, J., Lewinsohn, P., Rohde, P., Seeley, J., Monson, C.M., Meyer, K.A. \& Langford, R. (1998). Gender Differences in the Suicide-Related Behaviors of Adolescents and Young Adults, Sex Roles 39(11-12), p. 839-854.

LeBouthillier D.M., McMillan K.A., Thibodeau M.A., \& Asmundson G.J. (2015) Types and number of traumas associated with suicidal ideation and suicide attempts in PTSD: findings from a U.S. nationally representative sample. Journal of Trauma Stress 28(3), pp. 183-190

MacCallum, R.C., Browne, M.W., \& Sugawara, H.M. (1996). Power Analysis and Determination of Sample Size for Covariance Structure Modeling Psychological Methods, 1(2), pp. 130-149. Retrieved from: ftp://maia2.biostat.wisc.edu/pub/lindstrom/bakken/met-1-2-130.pdf

Marshall, R.D., Olfson, M., Hellman, F., Blanco, C., Guardino, M., \& Struening E.L. (2001). Comorbidity, Impairment, and Suicidality in Subthreshold PTSD. American Journal of Psychiatry, 158(9), pp. 1467-1473. https://doi.org/10.1176/appi.ajp.158.9.1467

Marmar, C.R., McCaslin, S.E., Metzler, T.J., Best, S., Weiss, D.S., Fagan, J.A., Liberman, K., Pole, N., Otte, C., Yehuda, R. Mohr, D., \& Neylan, T. (2006) 
Predictors of Posttraumatic Stress in Police and Other First Responders. Annals of New York Academy Sciences 1077, pp. 1-18. DOI: 10.1196/annals.1364.001

Marmar, C.R., Weiss, D.S., Metzler, T.J., Ronfeldt, H.M. \& Foreman, C. (1996). Stress responses of emergency services personnel to the Loma Prieta earthquake Interstate 880 freeway collapse and control traumatic incidents. J. Trauma. Stress 9: $63-85$

Martin, C.E. Tran, J.K., Buser, S.J. (2017). Correlates of suicidality in firefighter/EMS personnel, Journal of Affective Disorders, 208, pp. 177-183. https://doi.org/10.1016/j.jad.2016.08.078.

Mazerolle, P. (1998) Gender, General Strain, and Delinquency: An Empirical Examination. Justice Quarterly, 15(1) pp. 67-94

Mazerolle, P., Piquero, A.R., \& Capowich G.E. (2003) Examining the links between strain, situational and dispositional anger, and crime: Further specifying and testing general strain theory. Youth \& Society, 35, pp. 131-157

McCutcheon, V.V. Heath, A.C., Nelson, E.C., Bucholz, K.K., Madden, P.A.F., \& Martin, N.G. (2010) Clustering of Trauma and Associations with Single and CoOccurring Depression and Panic Attack over Twenty Years. Twin Research and Human Genetics, 13(1), pp. 57-65. https://doiorg.echo.louisville.edu/10.1375/twin.13.1.57

McKinney, J.M., Hirsch, J.K., \& Britton, P.C. (2017). PTSD symptoms and suicide risk in veterans: Serial indirect effects via depression and anger. Journal of Affective Disorders, 214, pp. 100-107 https://doi.org/10.1016/j.jad.2017.03.008 
McNally, R. J. and Robinaugh, D. J. (2011) Risk Factors and Posttraumatic Stress Disorder: Are they especially predictive following exposure to less severe stressors? Depression \& Anxiety, 28, pp. 1091-1096 DOI: 10.1002/da.20867

Ménard, K. S., \& Arter, M. L. (2013). Police Officer Alcohol Use and Trauma Symptoms: Associations with Critical Incidents, Coping, and Social Stressors. International Journal of Stress Management, 20(1), 37-56. doi:10.1037/a0031434

Merton, R.K. (1938) Social Structure and Anomie. American Sociological Review, 3, pp. $672-682$.

Miller-Graff, L.E., Scrafford, K., Rice, C. (2016) Conditional and indirect effects of age of first exposure on PTSD symptoms. Childhood Abuse and Neglect (51) pp. 303312 https://www-sciencedirectcom.echo.louisville.edu/science/article/pii/S014521341500318X?_rdoc=1\&_fmt= high \&_origin=gateway\&_docanchor=\&md5=b8429449ccfc9c30159a5f9aeaa92ff $\mathrm{b}$

Moon, B., Morash, M., McCluskey, C.P., \& Hwang, H.W. (2009) A Comprehensive Test of General Strain Theory: Key Strains, Situational and Trait-Based Negative Emotions, Conditioning Factors, and Delinquency. Journal of Research in Crime and Delinquency, 46(2). DOI: 10.1177/0022427808330873

Moulden, H.M. \& Firestone, P.F. (2007). Vicarious Traumatization: The Impact on Therapists Who Work with Sexual Offenders. Trauma, Violence, \& Abuse, 8(1), pp. 67-83. DOI: $10.1177 / 1524838006297729$

Neupane, S.P., Bramness, J.G. \& Lien, L. (2017). Comorbid post-traumatic stress disorder in alcohol use disorder: relationships to demography, drinking and 
neuroimmune profile. BMC Psychiatry, 17(312) DOI: 10.1186/s12888-017-14798

Panagioti, M., Gooding, P. A., \& Tarrier, N. (2015). A Prospective Study of Suicidal Ideation in Posttraumatic Stress Disorder: The Role of Perceptions of Defeat and Entrapment. Journal of Clinical Psychology, 71(1), pp. 50-61.

Paternoster, R. \& Mazerolle, P. (1994) General Strain Theory and Delinquency: A Replication and Extension. Journal of Research in Crime and Delinquency, 31(3), pp. 235-263.

Panagioti, M., Gooding, P. A., \& Tarrier, N. (2015). A Prospective Study of Suicidal Ideation in Posttraumatic Stress Disorder: The Role of Perceptions of Defeat and Entrapment. Journal of Clinical Psychology, 71(1), pp. 50-61.

Panagioti, M., Gooding, P. A., Triantafyllou, K., \& Tarrier, N. (2015). Suicidality and posttraumatic stress disorder (PTSD) in adolescents: A systematic review and meta-analysis, Social Psychiatry and Psychiatric Epidemiology, 50(4), DOI:10.1007/s00127-014-0978-X

Poindexter, E.K., Mitchell, S.M., Jahnd, D.R., Smith, P.N., Hirsch, J.K., \& Cukrowicz, K.C. (2015). PTSD symptoms and suicide ideation: Testing the conditional indirect effects of thwarted interpersonal needs and using substances to cope. Personality and Individual Differences, 72, pp. 167-172

Raab, P.A.; Mackintosh, M.A.; Gros, D.; \& Morland, L. (2015). Impact of comorbid depression on quality of life in male combat Veterans with posttraumatic stress disorder. Journal of Rehabilitation Research \& Development, 52(5). dx.doi.org/10.1682/JRRD.2014.05.0130 
Regambal, M.J. \& Alden, L.E. (2009). Pathways to intrusive memories in a trauma analogue paradigm: a structural equation model. Depression and Anxiety 26(2), pp. 156-165. DOI: 10.1002/da.20483

Rebellan, C.J., Manasse, M.E., Van Gundy, K.T., \& Cohn, E.S. (2012) Perceived injustice and delinquency: A test of general strain theory. Journal of Criminal Justice, 40(3) pp. 230-237. https://doi.org/10.1016/j.jcrimjus.2012.02.001

Rebellan, C.J., Piquero, N.L., Piquero, A.R., \& Sherod, T. (2009) Do Frustrated Economic Expectations and Objective Economic Inequity Promote Crime? A Randomized Experiment Testing Agnew's General Strain Theory. European Journal of Criminology, 6(47). DOI: 10.1177/1477370808098105

Robinson, H.M., Sigman, M.R., \& Wilson, J.P. (1997). Duty-related Stressors and PTSD Symptoms in Suburban Police Officers. Psychological Reports, 81, pp. 835-845

Rojas, S.M., Bilsky, S.A., Dutton, C., Badour, C.L. Feldner, M.T., \& Leen-Feldner, E.W. (2017). Lifetime histories of PTSD, suicidal ideation, and suicide attempts in a nationally representative sample of adolescents: Examining indirect effects via the roles of family and peer social support. Journal of Anxiety Disorders 49, pp. 95 103 https://doi.org/10.1016/j.janxdis.2017.04.006

Rytwinski N.K., Scur M.D., Feeny N.C., \& Youngstrom E.A. (2013). The co-occurrence of major depressive disorder among individuals with posttraumatic stress disorder: A meta-analysis. Journal of Traumatic Stress, 26(3). http://dx.doi.org/10.1002/jts.21814

Schreiber, J.B., Nora, A., Stage, F.K., Barlow E.A., \& King, J. (2006) Reporting Structural Equation Modeling and Confirmatory Factor Analysis Results: A 
Review. The Journal of Educational Research, 99(6) pp. 323-337

http://www.jstor.org/stable/27548147

Sher, L. (2004). Recognizing posttraumatic stress disorder. QJM: An International Journal of Medicine 97(1).https://doi.org/10.1093/qjmed/hch003

Smith, B.C., Armelie, A.P., Boarts, J.M., Brazil, M., \& Delahanty, D.L. (2016). PTSD, Depression, and Substance Use in Relation to Suicidality Risk among Traumatized Minority Lesbian, Gay, and Bisexual Youth. Archives of Suicide Research, 20, pp. 80-93 DOI: 10.1080/13811118.2015.1004484

Stanley, I.H., Hom, M.A., Spencer-Thomas, S. \& Joiner, T.E. (2017). Examining anxiety sensitivity as a mediator of the association between PTSD symptoms and suicide risk among women firefighters. Journal of Anxiety Disorders, 50, pp. 94-102.

Spinaris, C.G., Denhof, M.D., \& Kellaway (2012). Post-traumatic Stress Disorder in United States Corrections Professionals. Desert Waters Correctional Outreach.

Stevens, D., Wilcox, H.C., MacKinnon, D.F., Mondimore, F.M., Schweizer, B., Jancic, D., Coryell, W.H., Weissman, M.M., Levinson, D.F., \& Potash, J.B. (2013). Posttraumatic Stress Disorder increases risk for suicide attempt in adults with recurrent Major Depression. Depression and Anxiety 30(10), pp. 940-946. DOI: $10.1002 /$ da. 22160

Stone, D., Simon, T., Fowler, K., Kegler, S., Yuan, K., Holland, K., Ivey-Stephenson, A., Crosby, A. (2018) Vital Signs: Trends in State Suicide Rates — United States, 1999-2016 and Circumstances Contributing to Suicide — 27 States, 2015. Morbidity and Mortality Weekly Report, 67(22), pp. 617-624. https://www.cdc.gov/mmwr/volumes/67/wr/mm6722a1.htm?s_cid=mm6722a1_w 
Swartz, K., French, A.T., \& Roberts, A. (2017). Examining the Prevalence and Correlates of PTSD among Kentucky Department of Corrections Staff

Tiesman, H.M., Hendricks, S.A., Bell, J.L. \& Amandus, H.A. (2010). Eleven years of occupational mortality in law enforcement: The census of fatal occupational injuries, 1992-2002, American Journal of Industrial Medicine, 53(9), pp.940-949

Violanti, J.M. (2004). The American Association of Suicidology: Predictors of Police Suicide Ideation, Suicide and Life-Threatening Behavior, 34(3), pp. 277-284.

Wang, Z., Inslicht, S.S., Metzler, T. Henn-Haase, C. McCaslin, S.E., Tong, H., Neylan, T.C. \& Marmar, C.R. (2000) A prospective study of predictors of depression symptoms in police, Psychiatry Research, 175(3), pp. 211-216. https://doi.org/10.1016/j.psychres.2008.11.010.

Wilcox, H.C., Storr, C.L., \& Breslau, N. (2009).

Posttraumatic stress disorder and suicide attempts in a community sample of urban American

young adults. Archives of General Psychiatry, 66, pp. 305-311.DOI: 10.1001/archgenpsychiatry.2008.557

Young, G., Lareau, C. \& Pierre, B. (2014). One quintillion ways to have PTSD comorbidity: Recommendations for the disordered DSM-5. Psychological Injury And Law, 7(1). DOI: 10.1007/s12207-014-9186

Zhang, J., \& Lester, D. (2008). Psychological Tensions Found in Suicide Notes: A Test for the Strain Theory of Suicide. Archives of Suicide Research, 12(1), 67-73. doi:10.1080/13811110701800962 


\section{CURRICULUM VITA}

NAME: William “Clay” Johnson

ADDRESS: Department of Criminal Justice 2301 South $3^{\text {rd }}$ Street, University of Louisville

Louisville, Kentucky, 40208

DOB: Fort Thomas, Kentucky - May 14, 1992

EDUCATION \& TRAINING: B.A.; Psychology

University of Louisville, 2010-2015

M.S., Criminal Justice University of Louisville 2016-2018 